\title{
Cauchy Fluxes Associated with Tensor Fields Having Divergence Measure
}

\author{
Marco Degiovanni, Alfredo Marzocchi \\ $\&$ Alessandro Musesti
}

Communicated by D. OwEN

\begin{abstract}
Cauchy fluxes induced by locally summable tensor fields with divergence measure are characterized. The equivalence between integral formulations involving subsets of finite perimeter and much more restricted classes of subsets is proved.
\end{abstract}

\section{Introduction}

Since the proof by Cauchy of his celebrated Stress Theorem, many attempts have been made in order to generalize his ideas and remove certain additional hypotheses which did not seem to be natural. For instance, Cauchy assumed the traction exerted at a given point on a generic material surface to depend a priori only on the point and the normal at the surface at that point (the Cauchy Postulate); moreover, he supposed the traction field to depend continuously upon the point itself.

In [12] it was proved that, under suitable conditions, the Cauchy Postulate could be deduced from the balance of linear momentum. Moreover, in $[9,8]$ it was shown that the linear dependence of the traction upon the normal for almost all points could be derived from the same balance law, thus avoiding the continuity condition. In [8] the notion of Cauchy flux was also introduced, which has changed the basic concept in this kind of analysis. The main idea was to replace the exerted traction by the resultant (called Cauchyflux) on the material surface, thus specifying properties on the resultant and possibly avoiding those on the traction field. Some years later, it became clear that this approach could be developed in the setting of geometric measure theory $[19,15,16]$ and that the whole question refers more to the abstract structure of a balance law than the specific case of the stress. We refer the reader to [18, Chapter III] for an exposition of the basic results in this direction. We also mention [6] for a proof of Cauchy's Stress Theorem based on a variational technique, rather than a measure-theoretic one. 
Parallel to these studies, but intimately related, are the investigations on the concepts of subbody and material surface. Apart from their general use in the axiomatic foundation of continuum mechanics $[2,10,11,13,14,18]$, the collection $\mathscr{S}$ of all material surfaces of a given continuous body $B$ appears as the natural domain of the Cauchy flux $Q$ and the balance law is classically formulated for any subbody $M$ of $B$.

Thus, in the case of a scalar flux, a first question is to characterize the functions (Cauchy fluxes) $Q: \mathscr{S} \rightarrow \mathbb{R}$ of the form

$$
Q(S)=\int_{S} \boldsymbol{q} \cdot \boldsymbol{n}_{S} d . \mathscr{H} \mathbb{b}^{n-1} \quad \text { for any material surface } S \text { of } B
$$

under the condition that $\boldsymbol{q}$ (the flux vector) belongs to some function class. A related problem is to show that the integral form of the balance law

$$
\int_{\partial M} \boldsymbol{q} \cdot \boldsymbol{n}^{M} d \mathscr{H} \mathscr{C}^{n-1}=\int_{M} b d \mathscr{B}^{n} \quad \text { for any subbody } M \text { of } B
$$

is equivalent to the distributional equation $\operatorname{div} \boldsymbol{q}=b$ (for a detailed study of this point, involving also boundary conditions, see [1]).

The approach has to be generalized, when unbounded $\boldsymbol{q}$ 's are considered. In [16] the case where $\boldsymbol{q}$ and $\operatorname{div} \boldsymbol{q}$ are in $L^{p}$ is treated. In this situation, $Q$ is naturally defined only for "almost all" material surfaces $S$ and also the balance law (1.1) can be formulated only for "almost all" subbodies $M$. In [16] these notions are precisely defined and the Cauchy fluxes associated with flux vectors in $L^{p}$ with divergence in $L^{p}$ are then characterized. Moreover, the equivalence between the integral form of the balance law, formulated for almost all subbodies, and the distributional form is proved without extra assumptions.

The main purpose of our paper is to characterize the Cauchy fluxes $Q$ 's associated with flux vectors $q$ 's in $L_{\text {loc }}^{1}$ with divergence measure (see Corollary 5.5 and Theorem 7.1). We still call such fluxes balanced Cauchy fluxes. This seems to be the highest level of generality in which the integral form of the balance law can be written, provided that the term $b d \mathscr{D}^{n}$ is replaced by a (signed) measure. For this purpose, more general notions of "almost all" are considered here, which allow larger exceptional subsets. Nevertheless, we are still able to prove the equivalence between the integral form of the balance law, formulated for almost all subbodies, and the distributional form (see Theorem 7.2). Moreover, if $Q$ is vector valued and the flux tensor $\mathrm{q}$ satisfies a suitable estimate involving momenta, then $\mathrm{q}(x)$ is symmetric for a.e. $x$ 's (see Theorem 8.3).

As we have already observed, exceptional subsets have to be considered for the domain of $Q$ and for the formulation of the integral balance law. On the other hand, there is a certain degree of arbitrariness in the definition of such domains. As in [16], the notion of subbody is introduced here through a system of axioms which may allow both very large classes (e.g., normalized subsets of finite perimeter) and more restricted classes (e.g., normalized subsets with piecewise Lipschitz boundary). We refer the reader to [16, Sect. 3] for a detailed discussion of this aspect. Our second purpose is to show that any suitable set function $Q_{0}$, which is only defined for almost all $(n-1)$-dimensional intervals parallel to coordinate subspaces, can be uniquely 
extended to a balanced Cauchy flux $Q$ defined for almost all material surfaces (see Theorem 6.1). Moreover, if the balance law (1.1) is true for almost all $n$-dimensional intervals, then the distributional form follows (see Theorem 7.2). Therefore, at least for the problems we treat here, the choice of the family of subbodies seems not to be so crucial: the behavior of balance laws and Cauchy fluxes on very general objects (such as subsets of finite perimeter and Borel subsets of their boundaries) is determined by that on $n$-intervals and their faces. Also, notions like "almost all $n$-intervals" are more transparent than "almost all subbodies". This alternative approach seems to be more in the spirit of $[12,9,8]$ (see, e.g., [12, Theorem 4] and [8, Theorem 8]). On the other hand, situations of this kind are typical in classical measure theory, where each set function, defined on $n$-intervals and satisfying suitable conditions, can be uniquely extended to a measure defined on all Borel subsets.

\section{Review of Measure Theory}

In this section we briefly recall some well known notions of geometric measure theory. For detailed expositions, the reader is referred to $[4,7,17,20]$.

If $M \subseteq \mathbb{R}^{n}$, we denote by $\operatorname{diam} M, \operatorname{cl} M$, int $M$ and bd $M$ the diameter, the closure, the interior and the boundary of $M$ in $\mathbb{R}^{n}$, respectively. We also denote by $\mathrm{B}_{r}(x)$ the open ball of center $x$ and radius $r$ and, when $M$ is a Borel set, by $\mathfrak{B}(M)$ the $\sigma$-algebra of Borel subsets of $M$.

We denote by $\mathscr{D}^{n}$ the Lebesgue outer measure and by $\mathscr{H} \mathscr{C}^{k}$ the $k$-dimensional Hausdorff outer measure. Then we denote by $M_{*}$ the set of $x$ 's in $\mathbb{R}^{n}$ such that

$$
\lim _{r \rightarrow 0^{+}} \frac{\mathscr{D}^{n}\left(\mathrm{~B}_{r}(x) \backslash M\right)}{\mathscr{D}^{n}\left(\mathrm{~B}_{r}(x)\right)}=0
$$

and by $\partial_{*} M$ the measure-theoretic boundary of $M$, i.e.,

$$
\partial_{*} M=\mathbb{R}^{n} \backslash\left(M_{*} \cup\left(\mathbb{R}^{n} \backslash M\right)_{*}\right) .
$$

It is well known that $M_{*}$ and $\partial_{*} M$ are Borel subsets of $\mathbb{R}^{n}$. We say that $M$ is normalized, if $M_{*}=M$.

Proposition 2.1. Let $M, N$ be two normalized subsets of $\mathbb{R}^{n}$. Then

$$
\begin{aligned}
& {\left[\left(\left(\partial_{*} M\right) \backslash N\right) \cup\left(\left(\partial_{*} N\right) \backslash M\right)\right] \backslash\left(\partial_{*} M \cap \partial_{*} N\right) } \\
& \subseteq \partial_{*}\left((M \cup N)_{*}\right) \subseteq\left(\left(\partial_{*} M\right) \backslash N\right) \cup\left(\left(\partial_{*} N\right) \backslash M\right), \\
&\left(N \cap \partial_{*} M\right) \cup\left(M \cap \partial_{*} N\right) \subseteq \partial_{*}(M \cap N) \\
& \subseteq\left(N \cap \partial_{*} M\right) \cup\left(M \cap \partial_{*} N\right) \cup\left(\partial_{*} M \cap \partial_{*} N\right), \\
& {\left[\left(\left(\partial_{*} M\right) \backslash N\right) \cup\left(M \cap \partial_{*} N\right)\right] \backslash\left(\partial_{*} M \cap \partial_{*} N\right) \subseteq \partial_{*}\left((M \backslash N)_{*}\right) } \\
& \subseteq\left(\left(\partial_{*} M\right) \backslash N\right) \cup\left(M \cap \partial_{*} N\right) .
\end{aligned}
$$


Proof. See [11, Lemma 3.2] and [16, Proposition 2.1].

Now let $M \subseteq \mathbb{R}^{n}, x \in \partial_{*} M$ and $u \in \mathbb{R}^{n}$. We say that $u$ is a unit exterior normal vector to $M$ at $x$, if $|u|=1$ and

$$
\begin{aligned}
& \lim _{r \rightarrow 0^{+}} \frac{\mathscr{B}^{n}\left(\left\{\xi \in \mathrm{B}_{r}(x) \cap M:(\xi-x) \cdot u>0\right\}\right)}{\mathscr{D}^{n}\left(\mathrm{~B}_{r}(x)\right)}=0, \\
& \lim _{r \rightarrow 0^{+}} \frac{\mathscr{D}^{n}\left(\left\{\xi \in \mathrm{B}_{r}(x) \backslash M:(\xi-x) \cdot u<0\right\}\right)}{\mathscr{D}^{n}\left(\mathrm{~B}_{r}(x)\right)}=0 .
\end{aligned}
$$

If $u$ and $v$ are two unit exterior normal vectors to $M$ at $x$, it is readily seen that $u=v$. We define a map $\boldsymbol{n}^{M}: \partial_{*} M \rightarrow \mathbb{R}^{n}$, setting $\boldsymbol{n}^{M}(x)$ equal to the unit exterior normal vector to $M$ at $x$, if it does exist, $\boldsymbol{n}^{M}(x)=0$ otherwise. The map $\boldsymbol{n}^{M}$ turns out to be a bounded Borel map. It is called the unit exterior normal to $M$.

We say that $M$ has finite perimeter, if $\mathscr{H}^{n-1}\left(\partial_{*} M\right)<+\infty$ (this implies the $\mathscr{B}^{n}$-measurability of $\left.M\right)$. In such a case, $\left|\boldsymbol{n}^{M}(x)\right|=1$ for $\mathscr{H}^{n-1}$-a.e. $x \in \partial_{*} M$ and the Gauss-Green Theorem

$$
\int_{M} \boldsymbol{v} \cdot \nabla f d \mathscr{B}^{n}=\int_{\partial_{*} M} f \boldsymbol{v} \cdot \boldsymbol{n}^{M} d \mathscr{H} \mathscr{B}^{n-1}-\int_{M} f \operatorname{div} \boldsymbol{v} d \mathscr{D}^{n}
$$

holds whenever $f: \mathbb{R}^{n} \rightarrow \mathbb{R}$ and $\boldsymbol{v}: \mathbb{R}^{n} \rightarrow \mathbb{R}^{n}$ are Lipschitz continuous with compact support (see, e.g., [4, Theorem 4.5.6]).

Now let $\Omega$ be an open subset of $\mathbb{R}^{n}$ and $X$ a finite dimensional normed space. We denote by $\mathscr{D}_{\text {loc, },}^{1}(\Omega)$ the set of Borel functions $f: \Omega \rightarrow[0,+\infty]$ with $\int_{K} f d \mathscr{B}^{n}<+\infty$ for any compact subset $K$ of $\Omega$ and by $\mathscr{D}_{\mathrm{loc}}^{1}(\Omega ; X)$ the set of Borel maps $v: \Omega \rightarrow X$ with $\int_{K}\|\boldsymbol{v}\| d \mathscr{B}^{n}<+\infty$ for any compact subset $K$ of $\Omega$. Let us point out that the elements of such spaces are really functions, not equivalence classes of functions. The corresponding quotient spaces are denoted by $L_{\text {loc }}^{1}(\Omega ; X)$, etc. We also denote by $\mathfrak{M}(\Omega)$ the set of positive Borel measures $\mu: \mathfrak{B}(\Omega) \rightarrow[0,+\infty]$ finite on compact subsets of $\Omega$.

Finally, an ordered orthonormal basis $\left(e_{1}, \ldots, e_{n}\right)$ in $\mathbb{R}^{n}$ is called a frame. A frame $\left(e_{1}, \ldots, e_{n}\right)$ is said to be positively oriented, if the determinant of the matrix with columns $e_{1}, \ldots, e_{n}$ is positive.

\section{Balanced Cauchy Fluxes}

In this section we recall, with some adaptations, the main notions of [16].

An oriented surface $S$ in $\mathbb{R}^{n}$ is a pair $\left(\widehat{S}, \boldsymbol{n}_{S}\right)$, where $\widehat{S}$ is a Borel subset of $\mathbb{R}^{n}$ and $\boldsymbol{n}_{S}: \widehat{S} \rightarrow \mathbb{R}^{n}$ is a Borel map such that there exists a normalized set $M \subseteq \mathbb{R}^{n}$ of finite perimeter with $\widehat{S} \subseteq \partial_{*} M$ and $\boldsymbol{n}_{S}=\boldsymbol{n}^{M}{ }_{\mid \widehat{S}}$. In this case, we say that $S$ is subordinated to $M$. We call $\boldsymbol{n}_{S}$ the normal to the surface $S$. We also denote by $-S$ the oriented surface $\left(\widehat{S},-\boldsymbol{n}_{S}\right)$, which is subordinated to $\left(\mathbb{R}^{n} \backslash M\right)_{*}$. If $S, T$ are two oriented surfaces, we write $S \subseteq T$ if $\widehat{S} \subseteq \widehat{T}$ and $\boldsymbol{n}_{T \mid \widehat{S}}=\boldsymbol{n}_{S}$. Two oriented surfaces $S$ and $T$ are said to be compatible, if there exists a normalized set $M \subseteq \mathbb{R}^{n}$ of finite perimeter with $\widehat{S} \cup \widehat{T} \subseteq \partial_{*} M, \boldsymbol{n}_{S}=\boldsymbol{n}^{M}{ }_{\mid}$and $\boldsymbol{n}_{T}=\boldsymbol{n}^{M}{ }_{\mid \widehat{T}}$. They are said to be 
disjoint if $\widehat{S} \cap \widehat{T}=\emptyset$. If $S$ and $T$ are two compatible oriented surfaces, we denote by $S \cup T$ the oriented surface $\left(\widehat{S} \cup \widehat{T}, \boldsymbol{n}_{S \cup T}\right)$ such that

$$
\boldsymbol{n}_{S \cup T}(x)=\left\{\begin{array}{l}
\boldsymbol{n}_{S}(x) \text { if } x \in \widehat{S}, \\
\boldsymbol{n}_{T}(x) \text { if } x \in \widehat{T} .
\end{array}\right.
$$

In the following, we shall sometimes identify $\widehat{S}$ with $S$ and we shall consider expressions like, e.g., " $S$ is compact", " $\mathscr{H} \mathscr{C}^{n-1}(S)$ " instead of " $\widehat{S}$ is compact", " $\mathscr{H} \mathscr{C}^{n-1}(\widehat{S})$ ". In the same spirit, if $S$ is an oriented surface and $T$ is a Borel subset of $\widehat{S}$, we also denote by $T$ the oriented surface $\left(T, \boldsymbol{n}_{S \mid T}\right)$, provided that the reference to $S$ is clear.

Definition 3.1. Let $B$ be a bounded normalized subset of $\mathbb{R}^{n}$ of finite perimeter and let $\mathscr{P}$ be a collection of normalized subsets of $B$ of finite perimeter. We say that $\mathscr{P}$ is a system of parts (or a system of subbodies) of $B$, if the following conditions are satisfied:

(a) $\emptyset, B \in \mathscr{P}$;

(b) if $M, N \in \mathscr{P}$, then $(M \cup N)_{*}, M \cap N,(M \backslash N)_{*} \in \mathscr{P}$;

(c) if $M \in \mathscr{P}$ and $H \subseteq \mathbb{R}^{n}$ is an open affine half-space, then $M \cap H \in \mathscr{P}$.

The elements of $\mathscr{P}$ are called parts (or subbodies) of $B$ and the pair $(B, \mathscr{P})$ is called a continuous body.

Definition 3.2. Let $S$ be an oriented surface. We say that $S$ is a material surface of the continuous body $(B, \mathscr{P})$, if $S$ is subordinated to some $M \in \mathscr{P}$. We denote by $\mathscr{S}$ the collection of the material surfaces of $(B, \mathscr{P})$.

Definition 3.3. Let $(B, \mathscr{P})$ be a continuous body. For any $h \in \mathscr{L}_{\mathrm{loc},+}^{1}$ (int $\left.B\right)$ and $\eta \in \mathfrak{M}$ (int $B$ ) we set

$$
\begin{aligned}
& \mathscr{P}_{h \eta}=\left\{M \in \mathscr{P}: \operatorname{cl} M \subseteq \operatorname{int} B, \int_{\partial_{*} M} h d \mathscr{H}^{n-1}<+\infty \text { and } \eta\left(\partial_{*} M\right)=0\right\}, \\
& \mathscr{S}_{h \eta}=\left\{S \in \mathscr{S}: S \text { is subordinated to some } M \in \mathscr{P}_{h \eta}\right\} .
\end{aligned}
$$

Definition 3.4. Let $(B, \mathscr{P})$ be a continuous body. Then

(i) given $\mathscr{D} \subseteq \mathscr{P}$, we say that $\mathscr{D}$ contains almost all of $\mathscr{P}$ if $\mathscr{P}_{h \eta} \subseteq \mathscr{D}$ for some $h \in \mathscr{D}_{\mathrm{loc},+}^{1}$ (int $B$ ) and $\eta \in \mathfrak{M}$ (int $B$ );

(ii) given $\mathscr{D} \subseteq \mathscr{S}$, we say that $\mathscr{D}$ contains almost all of $\mathscr{S}$ if $\mathscr{S}_{h \eta} \subseteq \mathscr{D}$ for some $h \in \mathscr{D}_{\mathrm{loc},+}^{1}$ (int $B$ ) and $\eta \in \mathfrak{M}$ (int $B$ );

(iii) given a property $\pi$, we say that $\pi$ holds almost everywhere in $\mathscr{P}$ if the set

$$
\{M \in \mathscr{P}: \pi(M) \text { is defined and } \pi(M) \text { holds }\}
$$

contains almost all of $\mathscr{P}$; we say that $\pi$ holds almost everywhere in $\mathscr{S}$ if the set

$$
\{S \in \mathscr{S}: \pi(S) \text { is defined and } \pi(S) \text { holds }\}
$$

contains almost all of $\mathscr{S}$. 
Remark 3.5. No generality is lost in Definition 3.3 if we assume that $h: \operatorname{int} B \rightarrow$ $[0,+\infty]$ is a Borel function with $\int_{\text {int } B} h d \mathscr{B}^{n}<+\infty$ and $\eta: \mathfrak{B}$ (int $\left.B\right) \rightarrow$ $[0,+\infty]$ is a positive Borel measure with $\eta($ int $B)<+\infty$. In fact, given an increasing sequence $\left(K_{m}\right)$ of compact subsets of int $B$ with int $B=\bigcup_{m=1}^{\infty}$ int $K_{m}$, we may set

$$
\begin{gathered}
\hat{h}(x)= \begin{cases}\frac{h(x)}{1+\int_{K_{1}} h d \mathscr{B}^{n}} & \text { if } x \in K_{1}, \\
\frac{h(x)}{2^{m-1}\left(1+\int_{K_{m}} h d \mathscr{B}^{n}\right)} & \text { if } x \in K_{m} \backslash K_{m-1}, m \geqq 2,\end{cases} \\
\left.\hat{\eta}(M)=\frac{\eta\left(M \cap K_{1}\right)}{1+\eta\left(K_{1}\right)}+\sum_{m=2}^{\infty} \frac{\eta\left(M \cap\left(K_{m} \backslash K_{m-1}\right)\right)}{2^{m-1}\left(1+\eta\left(K_{m}\right)\right)} \quad(M \in \mathfrak{B} \text { (int } B)\right) .
\end{gathered}
$$

Then $\hat{h}, \hat{\eta}$ have the required properties and $\mathscr{P}_{\hat{h} \hat{\eta}}=\mathscr{P}_{h \eta}, \mathscr{S}_{\hat{h} \hat{\eta}}=\mathscr{S}_{h \eta}$.

Remark 3.6. Definition 3.4 should be compared with [16, Definition 4.1]. There only the case $\eta=0$ was considered. Moreover, $h$ was defined on $B$ with $\int_{B} h d \mathscr{B}^{n}<$ $+\infty$, but bd $B$ was assumed to be $\mathscr{D}^{n}$-negligible. By the previous remark, this last difference is inessential if attention is restricted to the case $\mathscr{L}^{n}(\mathrm{bd} B)=0$. The main point is that we want to consider vector fields $\boldsymbol{q}$ whose distributional divergence is a measure and to prove a Gauss-Green formula like

$$
\int_{\partial_{*} M} \boldsymbol{q} \cdot \boldsymbol{n}^{M} d \mathscr{H} \mathscr{C}^{n-1}=\int_{M} \operatorname{div} \boldsymbol{q}
$$

for almost every $M \in \mathscr{P}$. To expect such a formula, it seems to be necessary to impose the condition $|\operatorname{div} \boldsymbol{q}|\left(\partial_{*} M\right)=0$, which is automatically satisfied when $|\operatorname{div} \boldsymbol{q}|$ is absolutely continuous with respect to $\mathscr{B}^{n}$. On the other hand, it is not so restrictive to require that $\eta\left(\partial_{*} M\right)=0$ : Since $\eta$ is finite on compact subsets of int $B$, there are "not so many" Borel sets $S$ with $\mathscr{H}^{n-1}(S)<+\infty$ and $\eta(S)>0$.

Proposition 3.7. The following assertions hold:

(a) If $h \in \mathscr{D}_{\text {loc, }+}^{1}$ (int $\left.B\right), \eta \in \mathfrak{M}$ (int $\left.B\right)$ and $M_{1}, M_{2} \in \mathscr{P}_{h \eta}$, then $\left(M_{1} \cup M_{2}\right)_{*}$, $M_{1} \cap M_{2},\left(M_{1} \backslash M_{2}\right)_{*} \in \mathscr{P}_{h \eta}$.

(b) If $h \in \mathscr{L}_{\text {loc, }+}^{1}$ (int $B$ ), $\eta \in \mathfrak{M}$ (int $B$ ), $S, S_{1}, S_{2} \in \mathscr{S}_{h \eta}, T$ is an oriented surface with $T \subseteq S$ and $S_{1}, S_{2}$ are compatible, then $T, S_{1} \cup S_{2} \in \mathscr{S}_{h \eta}$.

(c) If $\left(h_{m}\right)$ is a sequence in $\mathscr{B}_{\mathrm{loc},+}^{1}$ (int $B$ ) and $\left(\eta_{m}\right)$ is a sequence in $\mathfrak{M}$ (int $B$ ), then there exist $h \in \mathscr{L}_{\mathrm{loc},+}^{1}$ (int $B$ ) and $\eta \in \mathfrak{M}$ (int $B$ ) such that

$$
\mathscr{P}_{h \eta} \subseteq \bigcap_{m=1}^{\infty} \mathscr{P}_{h_{m} \eta_{m}} \text { and } \mathscr{S}_{h \eta} \subseteq \bigcap_{m=1}^{\infty} \mathscr{S}_{h_{m} \eta_{m}} .
$$


Proof. Assertion (a) is a simple consequence of Proposition 2.1, while (b) is obvious.

To prove (c), consider an increasing sequence $\left(K_{m}\right)$ of compact subsets of int $B$ with int $B=\bigcup_{m=1}^{\infty}$ int $K_{m}$. It is readily seen that

$$
\begin{aligned}
h(x) & =\sum_{m=1}^{\infty} \frac{h_{m}(x)}{2^{m}\left(1+\int_{K_{m}} h_{m} d \mathscr{B}^{n}\right)}, \\
\eta(M) & =\sum_{m=1}^{\infty} \frac{\eta_{m}(M)}{2^{m} \eta_{m}\left(K_{m}\right)} \quad(M \in \mathfrak{B}(\operatorname{int} B))
\end{aligned}
$$

have the required properties.

The next result shows that the measure $\eta$ is effective only when it is "quite concentrated".

Proposition 3.8. Let $h \in \mathscr{D}_{\mathrm{loc},+}^{1}$ (int $\left.B\right)$ and $\eta \in \mathfrak{M}$ (int $B$ ). Assume that $\eta(T)=0$ whenever $T \in \mathfrak{B}$ (int $B$ ) and $\mathscr{H} \mathscr{C}^{n-1}(T)=0$. Then there exists $k \in \mathscr{L}_{\text {loc, }+}^{1}$ (int $B$ ) such that $\mathscr{P}_{k 0} \subseteq \mathscr{P}_{h \eta}$.

Proof. Let $\eta_{s}$ be the singular part of $\eta$ with respect to $\mathscr{B}^{n}$ and let $E$ be a Borel subset of int $B$ such that $\mathscr{B}^{n}(E)=0$ and $\eta_{s}(T)=\eta(T \cap E)$ for any $T \in \mathfrak{B}$ (int $B$ ). Set

$$
k(x)=\left\{\begin{array}{l}
h(x) \text { if } x \notin E, \\
+\infty \text { if } x \in E .
\end{array}\right.
$$

Then $k \in \mathscr{D}_{\text {loc, }+}^{1}$ (int $\left.B\right)$. Moreover, if $M \in \mathscr{P}_{k 0}$, we have $\mathscr{H}^{n-1}\left(E \cap \partial_{*} M\right)=0$. It follows $\eta_{s}\left(\partial_{*} M\right)=0$, hence $\eta\left(\partial_{*} M\right)=0$. Therefore $M \in \mathscr{P}_{h \eta}$.

Now we introduce one of the main notions of the paper.

Definition 3.9. Let $(B, \mathscr{P})$ be a continuous body, let $\mathscr{D} \subseteq \mathscr{S}$ be a set containing almost all of $\mathscr{S}$ and let $Q: \mathscr{D} \rightarrow \mathbb{R}$. We say that $Q$ is a (scalar) balanced Cauchy flux on $(B, \mathscr{P})$, if the following properties hold:

(a) If $S, T \in \mathscr{D}$ are compatible and disjoint with $S \cup T \in \mathscr{D}$, then

$$
Q(S \cup T)=Q(S)+Q(T) .
$$

(b) There exists $h \in \mathscr{L}_{\text {loc, }+}^{1}$ (int $B$ ) such that the inequality

$$
|Q(S)| \leqq \int_{S} h d \mathscr{H} \mathscr{B}^{n-1}
$$

holds almost everywhere in $\mathscr{S}$.

(c) There exists $\eta \in \mathfrak{M}$ (int $B$ ) such that the inequality

$$
\left|Q\left(\partial_{*} M\right)\right| \leqq \eta(M)
$$

holds almost everywhere in $\mathscr{P}$. 
Remark 3.10. In [16, Definition 5.1] a smaller class of balanced Cauchy fluxes (called of class $L^{p}$ ) was considered. As we have already observed, our notion of "almost everywhere" allows larger exceptional subsets of $\mathscr{P}$ and $\mathscr{S}$. Moreover, in [16] the $\mathscr{D}^{n}$-equivalence class of $h$ was supposed to be in $L^{p}$ (int $B$ ) and $\eta$ was assumed to be of the form $d \eta=k d \mathscr{B}^{n}$ with $k \in L^{p}$ (int $B$ ).

\section{A Uniqueness Criterion}

Throughout this section, $(B, \mathscr{P})$ denotes a continuous body.

Definition 4.1. A grid $G$ is an ordered triple

$$
G=\left(x_{0},\left(e_{1}, \ldots, e_{n}\right), \widehat{G}\right),
$$

where $x_{0} \in \mathbb{R}^{n},\left(e_{1}, \ldots, e_{n}\right)$ is a positively oriented frame in $\mathbb{R}^{n}$ and $\widehat{G}$ is a Borel subset of $\mathbb{R}$. If $G_{1} G_{2}$ are two grids, we write $G_{1} \subseteq G_{2}$ if the first two components coincide and $\widehat{G}_{1} \subseteq \widehat{G}_{2}$. A grid $G$ is said to be full, if $\mathscr{D}^{1}(\mathbb{R} \backslash \widehat{G})=0$. if

Let $G$ be a grid. A subset $I$ of $\mathbb{R}^{n}$ is said to be an open $n$-dimensional $G$-interval,

$$
I=\left\{x \in \mathbb{R}^{n}: \quad a^{(j)}<\left(x-x_{0}\right) \cdot e_{j}<b^{(j)} \quad \forall j=1, \ldots, n\right\}
$$

for some $a^{(1)}, b^{(1)}, \ldots, a^{(n)}, b^{(n)} \in \widehat{G}$. A subset $Y$ of $\mathbb{R}^{n}$ is said to be $a G$-figure, if $Y=\left(\bigcup_{I \in \mathscr{F}} I\right)_{*}$, where $\mathscr{F}$ is a finite family of open $n$-dimensional $G$-intervals.

Definition 4.2. For any grid $G$, we set

$$
\begin{aligned}
& \mathscr{T}_{G}=\{I: I \text { is an open } n \text {-dimensional } G \text {-interval with cl } I \subseteq \operatorname{int} B\}, \\
& \mathscr{T}_{G}=\{Y: Y \text { is a } G \text {-figure with } \operatorname{cl} Y \subseteq \operatorname{int} B\} .
\end{aligned}
$$

For $1 \leqq j \leqq n$, we also denote by $\mathscr{T}_{G}^{j}$ the family of all the oriented surfaces $S$ with

$$
\begin{aligned}
\widehat{S} & =\left\{x \in \mathbb{R}^{n}:\left(x-x_{0}\right) \cdot e_{j}=s, a^{(i)}<\left(x-x_{0}\right) \cdot e_{i}<b^{(i)} \forall i \neq j\right\}, \\
\boldsymbol{n}_{S} & =e_{j},
\end{aligned}
$$

$s, a^{(1)}, b^{(1)}, \ldots, a^{(j-1)}, b^{(j-1)}, a^{(j+1)}, b^{(j+1)}, \ldots, a^{(n)}, b^{(n)} \in \widehat{G}$ and

$\operatorname{cl} \widehat{S} \subseteq$ int $B$. Finally, we set

$$
\mathscr{T}_{G}=\bigcup_{j=1}^{n} \mathscr{T}_{G}^{j}
$$

Definition 4.3. Let $x_{0} \in \mathbb{R}^{n},\left(e_{1}, \ldots, e_{n}\right)$ be a positively oriented frame in $\mathbb{R}^{n}$, $E \subseteq \mathbb{R}^{n}$ and $1 \leqq j \leqq n$. For every $s \in \mathbb{R}$ we set

$$
\sigma_{j, s}(E)=\left\{x \in E:\left(x-x_{0}\right) \cdot e_{j}=s\right\} .
$$

If $G$ is a grid of the form $G=\left(x_{0},\left(e_{1}, \ldots, e_{n}\right), \widehat{G}\right), I \in \mathscr{T}_{G}$ and $s \in \widehat{G}$, we consider $\sigma_{j, s}(I)$ as an element of $\mathscr{S}_{G}^{j}$ with normal $\boldsymbol{n}_{\sigma_{j, s}(I)}=e_{j}$. 
Remark 4.4. For any grid $G$, we have $\mathscr{P}_{G} \subseteq \mathscr{P}$ and $\mathscr{S}_{G} \subseteq \mathscr{S}$.

Proposition 4.5. Let $x_{0} \in \mathbb{R}^{n}$ and $\left(e_{1}, \ldots, e_{n}\right)$ be a positively oriented frame in $\mathbb{R}^{n}$. Then for every $h \in \mathscr{D}_{\mathrm{loc},+}^{1}$ (int $B$ ) and $\eta \in \mathfrak{M}$ (int $B$ ) there exists a full grid $G$ of the form $G=\left(x_{0},\left(e_{1}, \ldots, e_{n}\right), \widehat{G}\right)$ such that $\mathscr{P}_{G} \subseteq \mathscr{P}_{h \eta}$ and $\mathscr{S}_{G} \subseteq \mathscr{S}_{h \eta}$.

Proof. Let $\left(K_{m}\right)$ be an increasing sequence of compact subsets of int $B$ with int $B=\bigcup_{m=1}^{\infty}$ int $K_{m}$. We have

$$
\forall m \in \mathbb{N}: \int_{K_{m}} h d \mathscr{B}^{n}<+\infty .
$$

Therefore, if we set

$$
D_{1}=\mathbb{R} \backslash\left(\bigcup_{m \in \mathbb{N}} \bigcup_{j=1}^{n}\left\{s \in \mathbb{R}: \int_{\sigma_{j, s}\left(K_{m}\right)} h d \mathscr{\mathscr { C }} \mathscr{C}^{n-1}=+\infty\right\}\right),
$$

it turns out that $D_{1}$ is a Borel set with $\mathscr{B}^{1}\left(\mathbb{R} \backslash D_{1}\right)=0$ by Fubini's Theorem.

On the other hand, we have $\eta\left(K_{m}\right)<+\infty$ for every $m \in \mathbb{N}$; hence $\eta\left(\sigma_{j, s}\left(K_{m}\right)\right)$ $\neq 0$ only for $s$ in a countable subset of $\mathbb{R}$. If we set

$$
D_{2}=\mathbb{R} \backslash\left(\bigcup_{j=1}^{n}\left\{s \in \mathbb{R}: \eta\left(\sigma_{j, s}(\text { int } B)\right)>0\right\}\right),
$$

we have that $D_{2}$ is a Borel set with $\mathscr{B}^{1}\left(\mathbb{R} \backslash D_{2}\right)=0$. Now it is easy to see that $\widehat{G}=D_{1} \cap D_{2}$ defines a grid with the required properties.

Theorem 4.6. Let $Q$ be a balanced Cauchy flux on $(B, \mathscr{P})$, let $x_{0} \in \mathbb{R}^{n}$ and let $\left(e_{1}, \ldots, e_{n}\right)$ be a positively oriented frame in $\mathbb{R}^{n}$. Then there exist $h \in \mathscr{L}_{\text {loc, }+}^{1}$ (int $\left.B\right)$, $\eta \in \mathfrak{M}$ (int $B)$ and a full grid $G$ of the form $G=\left(x_{0},\left(e_{1}, \ldots, e_{n}\right), \widehat{G}\right)$ with the following properties:

(a) The domain of $Q$ contains $\mathscr{S}_{h \eta}$; in particular, $Q(S \cup T)=Q(S)+Q(T)$ whenever $S, T \in \mathscr{S}_{h \eta}$ are compatible and disjoint.

(b) $|Q(S)| \leqq \int_{S} h d \mathscr{H}^{n-1}$ for any $S \in \mathscr{S}_{h \eta}$.

(c) $\left|Q\left(\partial_{*} M\right)\right| \leqq \eta(M)$ for any $M \in \mathscr{P}_{h \eta}$.

(d) $\mathscr{P}_{G} \subseteq \mathscr{P}_{h \eta}$ and $\mathscr{T}_{G} \subseteq \mathscr{S}_{h \eta}$.

Proof. By Proposition 3.7 there exist $h$ and $\eta$ satisfying (a)-(c). Then, by Proposition 4.5 , there exists a grid $G$ with the required properties.

Theorem 4.7. Let $Q$ be a balanced Cauchy flux on $(B, \mathscr{P})$ and let $h, \eta$ be as in (a)-(c) of Theorem 4.6. Then the following assertions hold:

(a) If ( $\left.S_{k}\right)$ is an increasing sequence in $\mathscr{S}_{h \eta}, S \in \mathscr{S}_{h \eta}, S_{k} \subseteq S$ and

$$
\mathscr{H}^{n-1}\left(S \backslash\left(\bigcup_{k=1}^{\infty} S_{k}\right)\right)=0 \text {, }
$$


then

$$
\lim _{k} Q\left(S_{k}\right)=Q(S)
$$

(b) If ( $\left.S_{k}\right)$ is a decreasing sequence in $\mathscr{S}_{h \eta}, S \in \mathscr{S}_{h \eta}, S \subseteq S_{k}$ and

$$
\mathscr{H}^{n-1}\left(\left(\bigcap_{k=1}^{\infty} S_{k}\right) \backslash S\right)=0,
$$

then

$$
\lim _{k} Q\left(S_{k}\right)=Q(S)
$$

(c) If $\left(M_{k}\right)$ is a decreasing sequence in $\mathscr{P}_{h \eta}, M \in \mathscr{P}_{h \eta}, S \in \mathscr{S}_{h \eta}$ is subordinated to $M, S \subseteq M_{k}$ and

$$
S=\left(\bigcap_{k=1}^{\infty}\left(M_{k} \cup \partial_{*} M_{k}\right)\right) \cap\left(M \cup \partial_{*} M\right),
$$

then

$$
\lim _{k} Q\left(M \cap \partial_{*} M_{k}\right)=\lim _{k} Q\left(\left(M \cup \partial_{*} M\right) \cap \partial_{*} M_{k}\right)=-Q(S) .
$$

(d) If $S \in \mathscr{T}_{h \eta}$, then $-S \in \mathscr{S}_{h \eta}$ and $Q(-S)=-Q(S)$.

Proof. To prove (a), we observe that $S \backslash S_{k} \in \mathscr{S}_{h \eta}$ and

$$
\left|Q(S)-Q\left(S_{k}\right)\right|=\left|Q\left(S \backslash S_{k}\right)\right| \leqq \int_{S \backslash S_{k}} h d \mathscr{H}^{n-1} .
$$

Then the assertion follows from Lebesgue's Theorem. The proof of (b) is similar.

To prove (c), we set $R_{k}=\partial_{*}\left(M \cap M_{k}\right) \backslash\left[\left(M \cap \partial_{*} M_{k}\right) \cup S\right]$. From Proposition 2.1 it follows that $\partial_{*}\left(M \cap M_{k}\right)$ is the disjoint union of $M \cap \partial_{*} M_{k}, S$ and $R_{k}$ and that

$$
R_{k} \subseteq\left[\left(M_{k} \cup \partial_{*} M_{k}\right) \cap \partial_{*} M\right] \backslash S .
$$

Moreover, we have

$$
\begin{gathered}
\left|Q\left(M \cap \partial_{*} M_{k}\right)+Q(S)+Q\left(R_{k}\right)\right|=\left|Q\left(\partial_{*}\left(M \cap M_{k}\right)\right)\right| \leqq \eta\left(M \cap M_{k}\right), \\
Q\left(\left(M \cup \partial_{*} M\right) \cap \partial_{*} M_{k}\right)=Q\left(M \cap \partial_{*} M_{k}\right)+Q\left(\partial_{*} M \cap \partial_{*} M_{k}\right),
\end{gathered}
$$

where $\partial_{*} M \cap \partial_{*} M_{k}$ has the orientation induced by $\partial_{*} M_{k}$. Since $\left(M \cap M_{k}\right)$ is a decreasing sequence of Borel sets with $\eta\left(M \cap M_{k}\right)<+\infty$ and with empty intersection, we have

$$
\lim _{k}\left|Q\left(M \cap \partial_{*} M_{k}\right)+Q(S)+Q\left(R_{k}\right)\right|=0 .
$$


On the other hand,

$$
\begin{aligned}
\left|Q\left(R_{k}\right)\right|+\left|Q\left(\partial_{*} M \cap \partial_{*} M_{k}\right)\right| & \leqq \int_{R_{k}} h d . \mathscr{\mathscr { C } ^ { n - 1 }}+\int_{\partial_{*} M \cap \partial_{*} M_{k}} h d \cdot \mathscr{H} \mathscr{C}^{n-1} \\
& \leqq 2 \int_{\left[\left(M_{k} \cup \partial_{*} M_{k}\right) \cap \partial_{*} M\right] \backslash S} h d \mathscr{H} \mathscr{C}^{n-1} .
\end{aligned}
$$

Now, $\left(\left[\left(M_{k} \cup \partial_{*} M_{k}\right) \cap \partial_{*} M\right] \backslash S\right)$ is a decreasing sequence of Borel subsets of $\partial_{*} M$ with empty intersection. From Lebesgue's Theorem we deduce that

$$
\lim _{k} Q\left(R_{k}\right)=\lim _{k} Q\left(\partial_{*} M \cap \partial_{*} M_{k}\right)=0
$$

and assertion (c) follows.

To prove (d), consider $S \in \mathscr{S}_{h \eta}$ subordinated to $M \in \mathscr{P}_{h \eta}$. Let $G$ be a full grid as in (d) of Theorem 4.6. Since cl $M \subseteq$ int $B$, by an easy variant of [5, Chapter 5, Lemma 1] there exists $Y \in \mathscr{P}_{G}$ such that $\mathrm{cl} M \subseteq Y$. Then $(Y \backslash M)_{*} \in \mathscr{P}_{h \eta}$ by Proposition 3.7 and $-S$ is subordinated to $(Y \backslash M)_{*}$ by Proposition 2.1. It follows that $-S \in \mathscr{S}_{h \eta}$.

Now assume that $S$ is compact. Let $\left(Y_{k}\right)$ be a decreasing sequence in $\mathscr{P}_{G}$ with $S \subseteq Y_{k}, \operatorname{cl} Y_{k} \subseteq Y$ and

$$
S=\bigcap_{k=1}^{\infty} \mathrm{cl} Y_{k}
$$

Since cl $M \subseteq Y$, we have

$$
\left((Y \backslash M)_{*}\right) \cup \partial_{*}\left((Y \backslash M)_{*}\right)=(Y \backslash M) \cup \partial_{*} Y .
$$

Then from assertion (c) we deduce that

$$
\begin{aligned}
\lim _{k} Q\left(M \cap \partial_{*} Y_{k}\right) & =-Q(S), \\
\lim _{k} Q\left((Y \backslash M) \cap \partial_{*} Y_{k}\right) & =-Q(-S) .
\end{aligned}
$$

On the other hand, we have

$$
\left|Q\left(M \cap \partial_{*} Y_{k}\right)+Q\left((Y \backslash M) \cap \partial_{*} Y_{k}\right)\right|=\left|Q\left(\partial_{*} Y_{k}\right)\right| \leqq \eta\left(Y_{k}\right)
$$

with $\lim _{k} \eta\left(Y_{k}\right)=\eta(S)=0$. It follows that $-Q(S)=Q(-S)$.

Finally, consider the general case. Since $\mathscr{T}^{n-1}(S)<+\infty$, from $[4$, Theorem 2.2.2] it easily follows that

$$
S=S_{0} \cup\left(\bigcup_{k=1}^{\infty} T_{k}\right),
$$

where $\mathscr{H}^{n-1}\left(S_{0}\right)=0$ and $\left(T_{k}\right)$ is an increasing sequence of compact sets. If $T_{k}$ is endowed with the orientation induced by $S$, from assertion $(a)$ we deduce that $\lim _{k} Q\left( \pm T_{k}\right)=Q( \pm S)$. On the other hand, we have $Q\left(-T_{k}\right)=-Q\left(T_{k}\right)$ by the previous step. Then assertion (d) follows in its full generality. 
Now we prove our main uniqueness criterion for Cauchy fluxes.

Lemma 4.8. Let $Q_{1}, Q_{2}$ be two balanced Cauchy fluxes on $(B, \mathscr{P})$ and let $h \in$ $\mathscr{L}_{\text {loc, }+}^{1}$ (int $B$ ), $\eta \in \mathfrak{M}$ (int $B$ ) be such that

(a) the domains of $Q_{1}$ and $Q_{2}$ contain $\mathscr{T}_{\mathrm{h}}$,

(b) $\left|Q_{i}(S)\right| \leqq \int_{S} h d \mathscr{H} \mathscr{C}^{n-1}$ for any $i=1,2$ and $S \in \mathscr{h} h$.

Finally, let $S_{0} \in \mathscr{S}_{h \eta}$. If $Q_{1}(T)=Q_{2}(T)$ for every compact material surface $T \subseteq S_{0}$, then $Q_{1}\left(S_{0}\right)=Q_{2}\left(S_{0}\right)$.

Proof. As before, we have

$$
S_{0}=T_{0} \cup\left(\bigcup_{k=1}^{\infty} T_{k}\right),
$$

where $\mathscr{H} \mathscr{C}^{n-1}\left(T_{0}\right)=0$ and $\left(T_{k}\right), k \geqq 1$, is an increasing sequence of compact sets. From (a) of Theorem 4.7 we deduce that $\lim _{k} Q_{i}\left(T_{k}\right)=Q_{i}\left(S_{0}\right)$. On the other hand, $Q_{1}$ and $Q_{2}$ agree on $T_{k}$, whence the assertion.

Theorem 4.9. Let $Q_{1}, Q_{2}$ be two balanced Cauchy fluxes on $(B, \mathscr{P})$ and let $G$ be a full grid. Suppose that the domains of $Q_{1}, Q_{2}$ contain $\mathscr{T}_{G}$ and that $Q_{1}=Q_{2}$ on $\mathscr{T}_{G}$. Then $Q_{1}=Q_{2}$ on almost all of $\mathscr{S}$.

Proof. Without loss of generality, we may assume that $G$ also satisfies (d) of Theorem 4.6 for some $h \in \mathscr{D}_{\text {loc, }+}^{1}$ (int $B$ ) and $\eta \in \mathfrak{M}$ (int $B$ ) satisfying (a)-(c) of the same theorem both for $Q_{1}$ and $Q_{2}$. The proof proceeds in steps.

I) Let $S \in \mathscr{S}_{G}^{j}$ and let $T$ be a compact material surface with $T \subseteq S$. Let $\left(Y_{k}\right)$ be a decreasing sequence in $\mathscr{P}_{G}$ with $T \subseteq Y_{k}$ and

$$
T=\bigcap_{k=1}^{\infty} \mathrm{cl} Y_{k} .
$$

Then $S \cap Y_{k} \in \mathscr{S}_{h \eta}$ and from (a), (b) of Theorem 4.6 it readily follows that $Q_{1}\left(S \cap Y_{k}\right)=Q_{2}\left(S \cap Y_{k}\right)$. On the other hand, from Theorem 4.7 we deduce that $\lim _{k} Q_{i}\left(S \cap Y_{k}\right)=Q_{i}(T)$, whence $Q_{1}(T)=Q_{2}(T)$.

II) If $S \in \mathscr{S}_{G}^{j}$ and $T$ is a material surface with $T \subseteq S$, we deduce from the previous step and Lemma 4.8 that $Q_{1}(T)=Q_{2}(T)$.

III) Consider $Y \in \mathscr{P}_{G}$ and a material surface $S$ subordinated to $Y$. Since $S$ is a disjoint Borel union

$$
S=S_{0} \cup\left(\bigcup_{k=1}^{m} T_{k}\right),
$$

with $\mathscr{B}^{n-1}\left(S_{0}\right)=0$ and $T_{k}$ or $-T_{k}$ contained in some $S_{k} \in \mathscr{\mathscr { C }}_{G}^{j_{k}}$, from the previous step and Theorems 4.6 and 4.7 we deduce that $Q_{1}(S)=Q_{2}(S)$. 
IV) Consider $S \in \mathscr{S}_{h \eta}$ with $S$ compact. Let $S$ be subordinated to $M \in \mathscr{P}_{h \eta}$ and let $\left(Y_{k}\right)$ be a decreasing sequence in $\mathscr{P}_{G}$ with $S \subseteq Y_{k}$ and

$$
S=\bigcap_{k=1}^{\infty} \operatorname{cl} Y_{k}
$$

From Theorem 4.7 we deduce that

$$
\lim _{k} Q_{i}\left(M \cap \partial_{*} Y_{k}\right)=-Q_{i}(S) .
$$

On the other hand, by the previous step we have $Q_{1}\left(M \cap \partial_{*} Y_{k}\right)=Q_{2}\left(M \cap \partial_{*} Y_{k}\right)$, whence $Q_{1}(S)=Q_{2}(S)$.

V) Finally, let $S \in \mathscr{S}_{h \eta}$. Combining the previous step with Lemma 4.8, we deduce that $Q_{1}(S)=Q_{2}(S)$ and the proof is complete.

\section{Vector Fields with Divergence Measure}

Definition 5.1. Let $\Omega$ be an open subset of $\mathbb{R}^{n}$ and let $\boldsymbol{q} \in \mathscr{D}_{\text {loc }}^{1}\left(\Omega ; \mathbb{R}^{n}\right)$. We say that $\operatorname{div} \boldsymbol{q}$ is $a$ (local) measure on $\Omega$, if $\operatorname{div} \boldsymbol{q}$ is a distribution on $\Omega$ of order 0 . This means that for every compact subset $K$ of $\Omega$ there exists a constant $c_{K}$ such that

$$
\left|\int_{\Omega} \boldsymbol{q} \cdot \nabla f d \mathscr{B}^{n}\right| \leqq c_{K} \max _{K}|f|
$$

whenever $f \in C_{0}^{\infty}(\Omega)$ and supt $f \subseteq K$. In such a case, there exist $\mu \in \mathfrak{M}(\Omega)$ and a Borel function $u: \Omega \rightarrow \mathbb{R}$ such that $|u(x)|=1$ for $\mu$-a.e. $x \in \Omega$ and

$$
-\int_{\Omega} \boldsymbol{q} \cdot \nabla f d \mathscr{D}^{n}=\int_{\Omega} f u d \mu
$$

for any Lipschitz function $f: \Omega \rightarrow \mathbb{R}$ with compact support. It is well known that $\mu$ is uniquely determined, while $u$ is uniquely determined $\mu$-almost everywhere. We put $|\operatorname{div} \boldsymbol{q}|=\mu$. Finally, if $M \in \mathfrak{B}(\Omega)$ and $f: M \rightarrow \mathbb{R}$ is Borel and $\mu$-summable on $M$, we set

$$
\int_{M} f \operatorname{div} \boldsymbol{q}=\int_{M} f u d \mu
$$

Theorem 5.2. Let $\boldsymbol{q} \in \mathscr{D}_{\mathrm{loc}}^{1}\left(\Omega ; \mathbb{R}^{n}\right)$ be a vector field with divergence measure. Then there exist a sequence $\left(\boldsymbol{q}_{m}\right)$ in $C^{\infty}\left(\Omega ; \mathbb{R}^{n}\right)$ and $h \in \mathscr{D}_{\mathrm{loc},+}^{1}(\Omega)$ such that

$$
\begin{gathered}
\forall x \in \Omega: h(x)<+\infty \Longrightarrow \lim _{m} \boldsymbol{q}_{m}(x)=\boldsymbol{q}(x), \\
\forall m \in \mathbb{N}, \forall x \in \Omega:\left|\boldsymbol{q}_{m}(x)\right| \leqq h(x) \text { and }\left|\boldsymbol{q}_{m}(x)\right| \leqq \underset{\Omega}{\operatorname{ess} \sup }|\boldsymbol{q}|, \\
\lim _{m} \int_{M} f \operatorname{div} \boldsymbol{q}_{m} d \mathscr{D}^{n}=\int_{M_{*}} f \operatorname{div} \boldsymbol{q},
\end{gathered}
$$

whenever $f: \Omega \rightarrow \mathbb{R}$ is continuous and $M \in \mathfrak{B}(\Omega)$ has compact closure in $\Omega$, provided that $|\operatorname{div} \boldsymbol{q}|\left(\partial_{*} M\right)=0$. 
Proof. Let $\rho \in C_{0}^{\infty}\left(\mathbb{R}^{n}\right)$ be a nonnegative function with $\int \rho d \mathscr{B}^{n}=1$ and let $\rho_{m}(x)=m^{n} \rho(m x)$. Let also $\left(K_{m}\right)$ be an increasing sequence of compact subsets of $\Omega$ with $\Omega=\bigcup_{m=1}^{\infty}$ int $K_{m}$ and let $\vartheta_{m} \in C_{0}^{\infty}(\Omega)$ with $0 \leqq \vartheta_{m} \leqq 1$ on $\Omega$ and $\vartheta_{m}=1$ on $K_{m}$. If we set

$$
\boldsymbol{q}_{m}(x)=\int_{\Omega} \rho_{m}(x-y) \vartheta_{m}(y) \boldsymbol{q}(y) d \mathscr{L}^{n}(y),
$$

it is well known that $\left(\boldsymbol{q}_{m}\right)$ is a sequence in $C^{\infty}\left(\Omega ; \mathbb{R}^{n}\right)$ converging to $\boldsymbol{q}$ in $L_{\mathrm{loc}}^{1}\left(\Omega ; \mathbb{R}^{n}\right)$ and satisfying $\left|\boldsymbol{q}_{m}(x)\right| \leqq$ ess sup $|\boldsymbol{q}|$. According to [3, Theorem IV.9], there exist a $\Omega$

subsequence, we still denote by $\left(\boldsymbol{q}_{m}\right)$, and $h \in \mathscr{B}_{\text {loc.+ }}^{1}(\Omega)$ satisfying (5.1) and (5.2).

Now let $f$ and $M$ be as in the statement of (5.3). For any sufficiently large $m$, we have

$$
\begin{aligned}
\int_{M} f(x) & \operatorname{div} \boldsymbol{q}_{m}(x) d \mathscr{B}^{n}(x) \\
= & \int_{M} f(x)\left(\int_{\Omega} \boldsymbol{q}(y) \cdot\left(\nabla \rho_{m}\right)(x-y) d \mathscr{D}^{n}(y)\right) d \mathscr{B}^{n}(x) \\
= & \int_{M} f(x)\left(\int_{\Omega} \rho_{m}(x-y) \operatorname{div} \boldsymbol{q}(y)\right) d \mathscr{D}^{n}(x) \\
= & \int_{\Omega}\left(\int_{M} f(x) \rho_{m}(x-y) d \mathscr{D}^{n}(x)\right) \operatorname{div} \boldsymbol{q}(y) .
\end{aligned}
$$

Moreover, if $K$ is a compact subset of $\Omega$ with cl $M \subseteq$ int $K$, we also have

$$
\left|\int_{M} f(x) \rho_{m}(x-y) d \mathscr{D}^{n}(x)\right| \leqq\left(\max _{K}|f|\right) \chi_{K}(y)
$$

eventually as $m \rightarrow \infty$. Finally, it is easy to see that the integral on the left-hand side is convergent to $f(y)$ on $M_{*}$ and to 0 on $(\Omega \backslash M)_{*}$, as $m \rightarrow \infty$. From Lebesgue's Theorem, (5.3) follows.

Theorem 5.3. Let $\boldsymbol{q} \in \mathscr{D}_{\mathrm{loc}}^{1}\left(\Omega ; \mathbb{R}^{n}\right)$ and let $\eta \in \mathfrak{M}(\Omega)$. Assume there exists a full grid $G$ such that, for any open $n$-dimensional $G$-interval I with $\mathrm{cl} I \subseteq \Omega$, one has that $\boldsymbol{q}$ is $\mathscr{H}^{n-1}$-summable on $\partial_{*} I$ and

$$
\left|\int_{\partial_{*} I} \boldsymbol{q} \cdot \boldsymbol{n}^{I} d \cdot \mathscr{B}^{n-1}\right| \leqq \eta(I) .
$$

Then $\boldsymbol{q}$ is a vector field with divergence measure and $|\operatorname{div} \boldsymbol{q}| \leqq \eta$.

Proof. Let $G=\left(x_{0},\left(e_{1}, \ldots, e_{n}\right), \widehat{G}\right)$ be a full grid as in the hypothesis. For every $x \in \mathbb{R}^{n}$ we set

$$
|x|_{\infty}=\max _{1 \leqq j \leqq n}\left|x \cdot e_{j}\right|
$$

and we define a function $\rho: \mathbb{R}^{n} \rightarrow \mathbb{R}$ by

$$
\rho(x)=2^{-n}(n+1)\left(1-|x|_{\infty}\right)^{+} .
$$


Then $\rho$ is a positive Lipschitz function which is zero outside of $J=\left\{x \in \mathbb{R}^{n}\right.$ : $\left.|x|_{\infty}<1\right\}$ and satisfies $\int \rho d \mathscr{B}^{n}=1$. Set $\rho_{m}(x)=m^{n} \rho(m x)$.

Let $K_{m}, \vartheta_{m}$ be as in the proof of Theorem 5.2 and let $\boldsymbol{q}_{m}: \Omega \rightarrow \mathbb{R}^{n}$ be the function of class $C^{1}$ defined by

$$
\boldsymbol{q}_{m}(x)=\int_{\Omega} \rho_{m}(x-y) \vartheta_{m}(y) \boldsymbol{q}(y) d \mathscr{D}^{n}(y) .
$$

Then $\left(\boldsymbol{q}_{m}\right)$ is convergent to $\boldsymbol{q}$ in $L_{\text {loc }}^{1}\left(\Omega ; \mathbb{R}^{n}\right)$ and for any $x \in \Omega$ we have

$$
\operatorname{div} \boldsymbol{q}_{m}(x)=\int_{\Omega} \vartheta_{m}(y) \boldsymbol{q}(y) \cdot\left(\nabla \rho_{m}\right)(x-y) d \mathscr{b}^{n}(y)
$$

We have to show that

$$
\sup \left\{\left|\int_{\Omega} \boldsymbol{q} \cdot \nabla f d \mathscr{D}^{n}\right|: f \in C_{0}^{\infty}(\Omega), \operatorname{supt} f \subseteq \omega, \max _{\omega}|f| \leqq 1\right\} \leqq \eta(\omega)
$$

for every open set $\omega$ with compact closure in $\Omega$. Now, if $\omega$ is such an open set and $f \in C_{0}^{\infty}(\Omega)$ with supt $f \subseteq \omega$, we have

$$
\begin{aligned}
\left|\int_{\Omega} \boldsymbol{q} \cdot \nabla f d \mathscr{D}^{n}\right| & =\lim _{m}\left|\int_{\Omega} \boldsymbol{q}_{m} \cdot \nabla f d \mathscr{B}^{n}\right| \\
& \leqq \liminf _{m} \int_{\Omega}|f|\left|\operatorname{div} \boldsymbol{q}_{m}\right| d \mathscr{D}^{n} \\
& \leqq\left(\max _{\omega}|f|\right) \liminf _{m} \int_{\text {supt } f}\left|\operatorname{div} \boldsymbol{q}_{m}\right| d \mathscr{D}^{n}
\end{aligned}
$$

Therefore, if we set $C=\operatorname{supt} f$, it is sufficient to show that

$$
\liminf _{m} \int_{C}\left|\operatorname{div} \boldsymbol{q}_{m}\right| d \mathscr{B}^{n} \leqq \eta(\omega)
$$

For every $x \in \mathbb{R}^{n}$ and for every $t \in\left[0,2^{-n}(n+1) m^{n}\right.$, we have

$$
\left\{y \in \mathbb{R}^{n}: \rho_{m}(x-y)>t\right\}=x+J_{m, t}, \quad J_{m, t}=\frac{2^{-n}(n+1) m^{n}-t}{2^{-n}(n+1) m^{n+1}} J
$$

Assume that $m$ is large enough to ensure that $C+J_{m, 0} \subseteq \omega$ and that $\vartheta_{m}=1$ on $C+J_{m, 0}$. Then for every $x \in C$ it turns out that $x+J_{m, t}$ is an open $n$-dimensional $G$-interval with closure in $\Omega$ for $\mathscr{D}^{1}$-a.e. $t \in\left[0,2^{-n}(n+1) m^{n}[\right.$ and from the 
Coarea Formula (see, e.g., [4, Theorem 3.2.12]) we deduce that

$$
\begin{aligned}
\left|\operatorname{div} \boldsymbol{q}_{m}(x)\right| & \\
& =\left|\int_{\left(x+J_{m, 0}\right)} \boldsymbol{q}(y) \cdot \frac{\left(\nabla \rho_{m}\right)(x-y)}{\left|\left(\nabla \rho_{m}\right)(x-y)\right|}\right|\left(\nabla \rho_{m}\right)(x-y)\left|d \mathscr{B}^{n}(y)\right| \\
& =\left|\int_{0}^{2^{-n}(n+1) m^{n}} \int_{\partial_{*}\left(x+J_{m, t}\right)} \boldsymbol{q}(y) \cdot \frac{\left(\nabla \rho_{m}\right)(x-y)}{\left|\left(\nabla \rho_{m}\right)(x-y)\right|} d \mathscr{H}^{n-1}(y) d \mathscr{D}^{1}(t)\right| \\
& \leqq \int_{0}^{2^{-n}(n+1) m^{n}} \eta\left(x+J_{m, t}\right) d \mathscr{C}^{1}(t) \\
& =2^{-n}(n+1) m^{n} \int_{0}^{1} \eta\left(x+\frac{s}{m} J\right) d \mathscr{D}^{1}(s) .
\end{aligned}
$$

From Fubini's Theorem it follows that

$$
\begin{aligned}
\int_{C} \mid \operatorname{div} & \boldsymbol{q}_{m}(x) \mid d \mathscr{B}^{n}(x) \\
& \leqq 2^{-n}(n+1) m^{n} \int_{C} \int_{0}^{1} \eta\left(x+\frac{s}{m} J\right) d \mathscr{C}^{1}(s) d \mathscr{L}^{n}(x) \\
& =2^{-n}(n+1) m^{n} \int_{0}^{1} \int_{C} \int_{\omega} \chi_{x+\frac{s}{m}} J(y) d \eta(y) d \mathscr{B}^{n}(x) d \mathscr{B}^{1}(s) .
\end{aligned}
$$

Again from Fubini's Theorem we conclude that

$$
\begin{aligned}
\int_{C}\left|\operatorname{div} \boldsymbol{q}_{m}(x)\right| d \mathscr{L}^{n}(x) \\
\quad \leqq 2^{-n}(n+1) m^{n} \int_{0}^{1} \int_{\omega} \int_{C} \chi_{y+\frac{s}{m} J}(x) d \mathscr{B}^{n}(x) d \eta(y) d \mathscr{D}^{1}(s) \\
\quad \leqq 2^{-n}(n+1) m^{n} \int_{0}^{1} \int_{\omega} \frac{2^{n} s^{n}}{m^{n}} d \eta(y) d \mathscr{D}^{1}(s)=\eta(\omega) .
\end{aligned}
$$

Passing to the lower limit as $m \rightarrow \infty$ we obtain assertion (5.4).

Now let $(B, \mathscr{P})$ be a continuous body and let $\boldsymbol{q} \in \mathscr{D}_{\text {loc }}^{1}$ (int $\left.B ; \mathbb{R}^{n}\right)$ be a vector field with divergence measure. Let $h \in \mathscr{D}_{\mathrm{loc},+}^{1}$ (int $B$ ) be as in Theorem 5.2 and let $\eta=|\operatorname{div} \boldsymbol{q}|$.

Theorem 5.4. For every $M \in \mathscr{P}_{h \eta}$ and for every locally Lipschitz function $f$ : int $B \rightarrow \mathbb{R}$, the function $\boldsymbol{q}$ is $\mathscr{\mathscr { B } ^ { n - 1 }}$-summable on $\partial_{*} M$ and

$$
\int_{M} \boldsymbol{q} \cdot \nabla f d \mathscr{B}^{n}=\int_{\partial_{*} M} f \boldsymbol{q} \cdot \boldsymbol{n}^{M} d \mathscr{H} \mathscr{G}^{n-1}-\int_{M} f \operatorname{div} \boldsymbol{q} .
$$


Proof. Let $\left(\boldsymbol{q}_{m}\right)$ be as in Theorem 5.2. Also let $M$ and $f$ be as in the statement of the theorem. Since $\boldsymbol{q}$ is a Borel map satisfying $|\boldsymbol{q}| \leqq h$, it is plain that $\boldsymbol{q}$ is $\mathscr{H} \mathscr{b}^{n-1}$ summable on $\partial_{*} M$. Moreover, $f$ is Lipschitz continuous in a neighborhood of $\mathrm{cl} M$, so that

$$
\int_{M} \boldsymbol{q}_{m} \cdot \nabla f d \mathscr{D}^{n}=\int_{\partial_{*} M} f \boldsymbol{q}_{m} \cdot \boldsymbol{n}^{M} d \mathscr{\mathscr { H }} \mathscr{b}^{n-1}-\int_{M} f \operatorname{div} \boldsymbol{q}_{m} d \mathscr{D}^{n} .
$$

Now we pass to the limit as $m \rightarrow \infty$. We apply Lebesgue's Theorem to the first two integrals, while the third one passes to the limit by (5.3). Therefore the proof is complete.

Corollary 5.5. For every $S \in \mathscr{S}_{h \eta}$ the map $q$ is $\mathscr{H}^{n-1}$-summable on $S$ and the formula

$$
Q(S)=\int_{S} \boldsymbol{q} \cdot \boldsymbol{n}_{S} d \mathscr{H} \mathscr{B}^{n-1}
$$

defines a balanced Cauchy flux $Q: \mathscr{S}_{h \eta} \rightarrow \mathbb{R}$ on $(B, \mathscr{P})$.

Proof. Of course, $Q$ is well defined on $\mathscr{h} \eta$, which contains almost all of $\mathscr{S}$, and satisfies properties (a) and (b) of Definition 3.9. If $M \in \mathscr{R} \eta$ and we apply Theorem 5.4 with $f=1$, we get

$$
\left|Q\left(\partial_{*} M\right)\right|=\left|\int_{\partial_{*} M} \boldsymbol{q} \cdot \boldsymbol{n}^{M} d \mathscr{H} \mathscr{C}^{n-1}\right|=\left|\int_{M} \operatorname{div} \boldsymbol{q}\right| \leqq|\operatorname{div} \boldsymbol{q}|(M)=\eta(M) .
$$

Therefore (c) of Definition 3.9 also follows.

Proposition 5.6. Let $\check{\boldsymbol{q}} \in \mathscr{D}_{\mathrm{loc}}^{1}$ (int $B ; \mathbb{R}^{n}$ ) be another vector field with divergence measure and let $G$ be a full grid. Assume that $\boldsymbol{q}$ and $\check{\boldsymbol{q}}$ are both $\mathscr{H}^{n-1}$-summable on any $S \in \mathscr{S}_{G}$ and that

$$
\forall S \in \mathscr{T}_{G}: \int_{S} \boldsymbol{q} \cdot \boldsymbol{n}_{S} d \mathscr{H} \mathscr{C}^{n-1}=\int_{S} \check{\boldsymbol{q}} \cdot \boldsymbol{n}_{S} d \mathscr{\mathscr { H }} \mathscr{C}^{n-1} .
$$

Then $\boldsymbol{q}(x)=\check{\boldsymbol{q}}(x)$ for $\mathscr{D}^{n}$-a.e. $x \in$ int $B$.

Proof. From Fubini's Theorem, we deduce that for any $j=1, \ldots, n$ and any

$$
I=\left\{x \in \mathbb{R}^{n}: \quad a^{(i)}<\left(x-x_{0}\right) \cdot e_{i}<b^{(i)} \quad \forall i=1, \ldots, n\right\} \in \mathscr{T}_{G}
$$

we have

$$
\begin{aligned}
\int_{I} \boldsymbol{q}^{(j)} d \mathscr{D}^{n} & =\int_{a^{(j)}}^{b^{(j)}}\left[\int_{\sigma_{j, s}(I)} \boldsymbol{q}(x) \cdot e_{j} d \mathscr{H} \mathscr{C}^{n-1}(x)\right] d \mathscr{D}^{1}(s) \\
& =\int_{a^{(j)}}^{b^{(j)}}\left[\int_{\sigma_{j, s}(I)} \check{\boldsymbol{q}}(x) \cdot e_{j} d \mathscr{H} \mathscr{C}^{n-1}(x)\right] d \mathscr{D}^{1}(s)=\int_{I} \check{\boldsymbol{q}}^{(j)} d \mathscr{D}^{n} .
\end{aligned}
$$

Since each open subset of int $B$ is a countable disjoint union of elements of $\mathscr{T}_{G}$, up to an $\mathscr{D}^{n}$-negligible set, the assertion follows. 
Corollary 5.7. Let $\check{\boldsymbol{q}} \in \mathscr{D}_{\mathrm{loc}}^{1}$ (int $B ; \mathbb{R}^{n}$ ) be another vector field with divergence measure and let $Q, \check{Q}$ be associated with $\boldsymbol{q}, \check{\boldsymbol{q}}$, according to Corollary 5.5. Then $\boldsymbol{q}(x)=\check{\boldsymbol{q}}(x)$ for $\mathscr{D}^{n}$-a.e. $x \in$ int $B$ if and only if $Q=\check{Q}$ on almost all of $\mathscr{S}$.

Proof. Let $\check{h}$ be associated with $\check{\boldsymbol{q}}$, according to Theorem 5.2 and let $\check{\eta}=|\operatorname{div} \check{\boldsymbol{q}}|$. Assume first that $\boldsymbol{q}(x)=\check{\boldsymbol{q}}(x)$ for $\mathscr{D}^{n}$-a.e. $x \in$ int $B$. If we set $\bar{\eta}=\eta+\check{\eta}$ and

$$
\bar{h}(x)= \begin{cases}h(x)+\check{h}(x) & \text { if } \boldsymbol{q}(x)=\check{\boldsymbol{q}}(x), \\ +\infty & \text { if } \boldsymbol{q}(x) \neq \check{\boldsymbol{q}}(x),\end{cases}
$$

it is readily seen that $Q$ and $\check{Q}$ agree on $\mathscr{S}_{\bar{h}} \bar{\eta}$.

Now assume that $Q=\check{Q}$ on almost all of $\mathscr{S}$. Let $\bar{h} \in \mathscr{D}_{\text {loc, }+}^{1}$ (int $B$ ) and $\bar{\eta} \in \mathfrak{M}$ (int $B$ ) be such that $\mathscr{S}_{\bar{h} \bar{\eta}} \subseteq \mathscr{S}_{h \eta} \cap \mathscr{S}_{\bar{h} \check{\eta}}$ and such that $Q, \check{Q}$ agree on $\mathscr{T}_{\bar{h} \bar{\eta}}$. By Proposition 4.5 there exists a full grid $G$ with $\mathscr{S}_{G} \subseteq \mathscr{S}_{\bar{h}} \bar{\eta}$. From Proposition 5.6 we conclude that $\boldsymbol{q}(x)=\check{\boldsymbol{q}}(x)$ for $\mathscr{B}^{n}$-a.e. $x \in \operatorname{int} B$.

\section{An Integral Representation and Extension Result}

Throughout this section, $(B, \mathscr{P})$ denotes a continuous body,

$$
G_{0}=\left(x_{0},\left(e_{1}, \ldots, e_{n}\right), \widehat{G}_{0}\right)
$$

a full grid and $Q_{0}: \mathscr{T}_{G_{0}} \rightarrow \mathbb{R}$ a function satisfying the following properties:

(i) $Q_{0}(S)=Q_{0}\left(S_{1}\right)+Q_{0}\left(S_{2}\right)$ whenever $S, S_{1}, S_{2} \in \mathscr{S}_{G_{0}}, S_{1} \cap S_{2}=\emptyset$ and $\operatorname{cl} S=\operatorname{cl} S_{1} \cup \operatorname{cl} S_{2}$.

(ii) There exists $h \in \mathscr{D}_{\text {loc, }+}^{1}$ (int $B$ ) such that

$$
\left|Q_{0}(S)\right| \leqq \int_{S} h d . \mathscr{H} \mathfrak{B}^{n-1}
$$

for any $S \in \mathscr{S}_{G_{0}}$.

(iii) There exists $\eta \in \mathfrak{M}$ (int $B$ ) such that

$$
\left|\sum_{j=1}^{n}\left(Q_{0}\left(\varphi_{j}^{+}(I)\right)-Q_{0}\left(\varphi_{j}^{-}(I)\right)\right)\right| \leqq \eta(I)
$$

whenever

$$
\begin{aligned}
I & =\left\{x \in \mathbb{R}^{n}: a^{(j)}<\left(x-x_{0}\right) \cdot e_{j}<b^{(j)} \quad \forall j=1, \ldots, n\right\} \in \mathscr{T}_{G_{0}}, \\
\varphi_{j}^{+}(I)=\left\{x \in \mathbb{R}^{n}:\left(x-x_{0}\right) \cdot e_{j}=b^{(j)},\right. & \\
\left.a^{(i)}<\left(x-x_{0}\right) \cdot e_{i}<b^{(i)} \quad \forall i \neq j\right\}, & \\
\varphi_{j}^{-}(I)=\left\{x \in \mathbb{R}^{n}:\left(x-x_{0}\right) \cdot e_{j}=a^{(j)},\right. & \\
a^{(i)} & \left.<\left(x-x_{0}\right) \cdot e_{i}<b^{(i)} \quad \forall i \neq j\right\} .
\end{aligned}
$$


Although the domain of $Q_{0}$ is quite restricted, assumptions (ii) and (iii) provide such a uniform control that $Q_{0}$ can be uniquely extended to almost all of $\mathscr{S}$, as the next theorem shows.

Theorem 6.1. There exist a balanced Cauchy flux $Q$ on $(B, \mathscr{P})$, a vector field $\boldsymbol{q} \in \mathscr{D}_{\text {loc }}^{1}$ (int $B ; \mathbb{R}^{n}$ ) with divergence measure and a full grid $G \subseteq G_{0}$ satisfying the following conditions:

(a) the domain of $Q$ contains $\mathscr{T}_{G}, \boldsymbol{q}$ is $\mathscr{H}^{n-1}$-summable on any $S \in \mathscr{T}_{G}$ and

$$
\forall S \in \mathscr{T}_{G}: Q(S)=\int_{S} \boldsymbol{q} \cdot \boldsymbol{n}_{S} d \cdot \mathscr{H} \mathscr{C}^{n-1}=Q_{0}(S) .
$$

(b) $|\operatorname{div} \boldsymbol{q}| \leqq \eta$ and

$$
Q(S)=\int_{S} \boldsymbol{q} \cdot \boldsymbol{n}_{S} d \mathscr{\mathscr { H }} \mathscr{C}^{n-1}
$$

on almost all of $\mathscr{S}$.

Moreover, if $\check{Q}$ and $\check{\boldsymbol{q}}$ also satisfy (a) for some full grid $\check{G} \subseteq G_{0}$, then $\check{Q}=Q$ on almost all of $\mathscr{S}$ and $\check{\boldsymbol{q}}(x)=\boldsymbol{q}(x)$ for $\mathscr{D}^{n}-$ a.e. $x \in$ int $B$.

The section is devoted to the proof of this result. First of all, let $h$ and $\eta$ be as in assumptions (ii) and (iii). By Proposition 4.5, we may suppose without loss of generality that $\mathscr{P}_{G_{0}} \subseteq \mathscr{P}_{h \eta}$ and $\mathscr{S}_{G_{0}} \subseteq \mathscr{S}_{h \eta}$. Moreover, for any

$$
I=\left\{x \in \mathbb{R}^{n}: \quad a^{(j)}<\left(x-x_{0}\right) \cdot e_{j}<b^{(j)} \quad \forall j=1, \ldots, n\right\} \in \mathscr{T}_{G_{0}}
$$

we may set

$$
Q_{0}\left(\partial_{*} I\right)=\sum_{j=1}^{n}\left(Q_{0}\left(\varphi_{j}^{+}(I)\right)-Q_{0}\left(\varphi_{j}^{-}(I)\right)\right),
$$

where $\varphi_{j}^{ \pm}(I)$ are defined as before.

Lemma 6.2. For every $j=1, \ldots, n$, the following assertions hold:

(a) If

$$
I=\left\{x \in \mathbb{R}^{n}: \quad a^{(i)}<\left(x-x_{0}\right) \cdot e_{i}<b^{(i)} \quad \forall i=1, \ldots, n\right\} \in \mathscr{T}_{G_{0}},
$$

then the function $\left\{s \mapsto Q_{0}\left(\sigma_{j, s}(I)\right)\right\}$ is continuous on $\left.\widehat{G}_{0} \cap\right] a^{(j)}, b^{(j)}[$ and any extension to $] a^{(j)}, b^{(j)}\left[\right.$ is $\mathscr{b}^{1}-$ summable.

(b) If $\mu_{j}: \mathscr{T}_{G_{0}} \rightarrow \mathbb{R}$ is defined by

$$
\mu_{j}(I)=\int_{a^{(j)}}^{b^{(j)}} Q_{0}\left(\sigma_{j, s}(I)\right) d \mathscr{D}^{1}(s)
$$

then

$$
\mu_{j}(I)=\mu_{j}\left(I_{1}\right)+\mu_{j}\left(I_{2}\right), \quad\left|\mu_{j}(J)\right| \leqq \int_{J} h d \mathscr{D}^{n}
$$


whenever $I, I_{1}, I_{2}, J \in \mathscr{T}_{G_{0}}, I_{1} \cap I_{2}=\emptyset, I_{1} \cup I_{2} \subseteq I$ and $\mathscr{S}^{n}\left(I \backslash\left(I_{1} \cup I_{2}\right)\right)=0$.

(c) There exists $\boldsymbol{q}^{(j)} \in \mathscr{D}_{\text {loc }}^{1}$ (int $B ; \mathbb{R}$ ) such that

$$
\mu_{j}(I)=\int_{I} \boldsymbol{q}^{(j)} d \mathscr{L}^{n}, \quad\left|\boldsymbol{q}^{(j)}(x)\right| \leqq h(x)
$$

for any $I \in \mathscr{T}_{G_{0}}$ and $x \in \operatorname{int} B$.

Proof. (a) We consider, for simplicity, the case $n=2$ and $j=1$. Let $r, s \in$ $\left.\widehat{G}_{0} \cap\right] a^{(1)}, b^{(1)}[$ with $r<s$. If

$$
I_{r}=\left\{x \in \mathbb{R}^{2}: r<\left(x-x_{0}\right) \cdot e_{1}<s, a^{(2)}<\left(x-x_{0}\right) \cdot e_{2}<b^{(2)}\right\},
$$

we have $I_{r} \in \mathscr{T}_{G_{0}}$, whence $\left|Q_{0}\left(\partial_{*} I_{r}\right)\right| \leqq \eta\left(I_{r}\right)$, and also $\lim _{r \rightarrow s^{-}} \eta\left(I_{r}\right)=0$. On the other hand, we have by definition

$$
Q_{0}\left(\partial_{*} I_{r}\right)=Q_{0}\left(\sigma_{1, s}(I)\right)-Q_{0}\left(\sigma_{1, r}(I)\right)+Q_{0}\left(\sigma_{2, b^{(2)}}\left(I_{r}\right)\right)-Q_{0}\left(\sigma_{2, a^{(2)}}\left(I_{r}\right)\right)
$$

and by assumption (ii)

$$
\begin{aligned}
\left|Q_{0}\left(\sigma_{2, a^{(2)}}\left(I_{r}\right)\right)\right| & \leqq \int_{\sigma_{2, a^{(2)}\left(I_{r}\right)}} h d \cdot \mathscr{H} \mathscr{C}^{1}(x), \\
\left|Q_{0}\left(\sigma_{2, b^{(2)}}\left(I_{r}\right)\right)\right| & \leqq \int_{\sigma_{2, b^{(2)}\left(I_{r}\right)}} h d \mathscr{H} \mathscr{C}^{1}(x) .
\end{aligned}
$$

It follows that

$$
\lim _{r \rightarrow s^{-}} Q_{0}\left(\sigma_{1, r}(I)\right)=Q_{0}\left(\sigma_{1, s}(I)\right) .
$$

The right continuity can be proved in a similar way.

Since $G$ is full, any extension of $\left\{s \longmapsto Q_{0}\left(\sigma_{1, s}(I)\right)\right\}$ to $] a^{(1)}, b^{(1)}\left[\right.$ is $\mathscr{B}^{1}$ measurable. Moreover, Fubini's Theorem and assumption (ii) yield

$$
\begin{aligned}
\int_{a^{(1)}}^{b^{(1)}}\left|Q_{0}\left(\sigma_{1, s}(I)\right)\right| d \mathscr{D}^{1}(s) & \leqq \int_{a^{(1)}}^{b^{(1)}}\left[\int_{\sigma_{1, s}(I)} h d \mathscr{\mathscr { C } ^ { 1 }}\right] d \mathscr{B}^{1}(s) \\
& =\int_{I} h d \mathscr{B}^{2}<+\infty
\end{aligned}
$$

whence the $\mathscr{B}^{1}$-summability of $\left\{s \longmapsto Q_{0}\left(\sigma_{1, s}(I)\right)\right\}$.

(b) The additivity is evident. If

$$
J=\left\{x \in \mathbb{R}^{n}: \quad c^{(i)}<\left(x-x_{0}\right) \cdot e_{i}<d^{(i)} \quad \forall i=1, \ldots, n\right\} \in \mathscr{T}_{G_{0}},
$$

we also have

$$
\left|\mu_{j}(J)\right|=\left|\int_{c^{(j)}}^{d^{(j)}} Q_{0}\left(\sigma_{j, s}(J)\right) d \mathscr{B}^{1}(s)\right| \leqq \int_{J} h d \mathscr{B}^{n} .
$$


(c) We may define a linear functional $T: C_{0}^{\infty}($ int $B) \rightarrow \mathbb{R}$ by

$$
\langle T, f\rangle=\lim \left(\sum_{m} f\left(\xi_{m}\right) \mu_{j}\left(I_{m}\right)\right) \quad \text { as } \sup _{m}\left(\operatorname{diam} I_{m}\right) \rightarrow 0,
$$

where each $\left\{I_{m}\right\}$ is a finite disjoint subfamily of $\mathscr{T}_{G_{0}}$ whose union contains supt $f$ up to an $\mathscr{D}^{n}$-negligible set and $\xi_{m} \in I_{m}$. It is readily seen that $T$ is a distribution of order 0 on int $B$ satisfying

$$
\forall f \in C_{0}^{\infty}(\text { int } B):|\langle T, f\rangle| \leqq \int_{\Omega}|f| h d \mathscr{B}^{n} .
$$

Combining the Riesz Representation Theorem with the Radon-Nikodym Theorem, we find $\boldsymbol{q}^{(j)}$ with the required properties.

Let $\boldsymbol{q} \in \mathscr{D}_{\text {loc }}^{1}$ (int $B ; \mathbb{R}^{n}$ ) be defined by

$$
\boldsymbol{q}(x)=\sum_{j=1}^{n} \boldsymbol{q}^{(j)}(x) e_{j}
$$

Lemma 6.3. There exists a full grid $G_{1} \subseteq G_{0}$ such that $\boldsymbol{q}$ is $\mathscr{H}^{n-1}$-summable on each $S \in \mathscr{G}_{G_{1}}$ and

$$
\begin{aligned}
& \forall S \in \mathscr{G}_{G_{1}}: Q_{0}(S) \quad=\int_{S} \boldsymbol{q} \cdot \boldsymbol{n}_{S} d \mathscr{H} \mathscr{C}^{n-1}, \\
& \forall I \in \mathscr{T}_{G_{1}}: Q_{0}\left(\partial_{*} I\right)=\int_{\partial_{*} I} \boldsymbol{q} \cdot \boldsymbol{n}^{I} d \mathscr{H} \mathscr{C}^{n-1} .
\end{aligned}
$$

Proof. Let $D_{2}$ be a countable and dense subset of $\widehat{G}_{0}$ and let $G_{2} \subseteq G_{0}$ be the grid such that $\widehat{G}_{2}=D_{2}$. Since $\mathscr{T}_{G_{2}}$ is countable, by Fubini's Theorem and well known results on Lebesgue points, there exists a full grid $G_{1} \subseteq G_{0}$ such that $\boldsymbol{q}$ is $\mathscr{H} \mathscr{G}^{n-1}$-summable on $\sigma_{j, s}(I)$ and

$$
\lim _{r \rightarrow 0^{+}} \frac{1}{2 r} \int_{s-r}^{s+r}\left|\int_{\sigma_{j, \tau}(I)} q_{j} d \mathscr{H} \mathscr{C}^{n-1}-\int_{\sigma_{j, s}(I)} q_{j} d \mathscr{H} \mathscr{C}^{n-1}\right| d \mathscr{D}^{1}(\tau)=0
$$

for any $I \in \mathscr{T}_{G_{2}}, 1 \leqq j \leqq n$ and $s \in \widehat{G}_{1}$. In particular, $\boldsymbol{q}$ is $\mathscr{H}^{n-1}$-summable on every compact subset of $\sigma_{j, s}$ (int $B$ ).

Now let $S \in \mathscr{S}_{G_{1}}^{j}$. First of all, we treat the particular case where

$$
\widehat{S}=\left\{x \in \mathbb{R}^{n}: \quad\left(x-x_{0}\right) \cdot e_{j}=s, \quad a^{(i)}<\left(x-x_{0}\right) \cdot e_{i}<b^{(i)} \quad \forall i \neq j\right\}
$$

with $s \in \widehat{G}_{1}$ and $a^{(1)}, b^{(1)}, \ldots, a^{(j-1)}, b^{(j-1)}, a^{(j+1)}, b^{(j+1)}, \ldots, a^{(n)}, b^{(n)} \in$ $D_{2}$. Let $a^{(j)}, b^{(j)} \in D_{2}$ be such that

$$
I=\left\{x \in \mathbb{R}^{n}: \quad a^{(i)}<\left(x-x_{0}\right) \cdot e_{i}<b^{(i)} \quad \forall i=1, \ldots, n\right\} \in \mathscr{T}_{G_{2}}
$$


and $a^{(j)}<s<b^{(j)}$. Finally, let $\left(r_{m}\right)$ be a sequence in $\widehat{G}_{0}$ strictly increasing to $s$ and $\left(s_{m}\right)$ a sequence in $\widehat{G}_{0}$ strictly decreasing to $s$. We have

$$
\begin{aligned}
& \frac{1}{s_{m}-r_{m}} \int_{r_{m}}^{s_{m}} Q_{0}\left(\sigma_{j, \tau}(I)\right) d \mathscr{D}^{1}(\tau) \\
& \quad=\frac{1}{s_{m}-r_{m}} \int_{r_{m}}^{s_{m}}\left[\int_{\sigma_{j, \tau}(I)} q_{j} d \mathscr{\mathscr { H }} \mathscr{C}^{n-1}\right] d \mathscr{D}^{1}(\tau) .
\end{aligned}
$$

Passing to the limit as $m \rightarrow \infty$ and taking into account (a) of Lemma 6.2, we deduce that

$$
Q_{0}(S)=\int_{S} q_{j} d . \mathscr{H} \mathscr{C}^{n-1} .
$$

Now consider a general $S \in \mathscr{S}_{G_{1}}^{j}$. Let

$$
\widehat{S}=\left\{x \in \mathbb{R}^{n}:\left(x-x_{0}\right) \cdot e_{j}=s, a^{(i)}<\left(x-x_{0}\right) \cdot e_{i}<b^{(i)} \quad \forall i \neq j\right\} .
$$

There exists an increasing sequence $\left(S_{m}\right)$ with

$$
\widehat{S}_{m}=\left\{x \in \mathbb{R}^{n}:\left(x-x_{0}\right) \cdot e_{j}=s, a_{m}^{(i)}<\left(x-x_{0}\right) \cdot e_{i}<b_{m}^{(i)} \quad \forall i \neq j\right\}
$$

whose union is $S$ with $a_{m}^{(i)}, b_{m}^{(i)} \in D_{2}$. From the previous step we have

$$
Q_{0}\left(S_{m}\right)=\int_{S_{m}} q_{j} d \mathscr{T} \mathscr{B}^{n-1} .
$$

On the other hand, it is easy to pass to the limit at the right-hand side, while the left-hand side also passes to the limit by assumptions (i) and (ii). Then the assertion follows.

The statement concerning $I \in \mathscr{T}_{G_{1}}$ is an obvious consequence. Therefore the proof is complete.

Proof of Theorem 6.1. We have already constructed a vector field $\boldsymbol{q} \in \mathscr{D}_{\text {loc }}^{1}$ (int $B$; $\mathbb{R}^{n}$ ) and we know from Lemma 6.3 that

$$
\begin{gathered}
\forall S \in \mathscr{T}_{G_{1}}: \quad Q_{0}(S)=\int_{S} \boldsymbol{q} \cdot \boldsymbol{n}_{S} d . \mathscr{H} \mathscr{C}^{n-1}, \\
\forall I \in \mathscr{T}_{G_{1}}: \quad\left|\int_{\partial_{*} I} \boldsymbol{q} \cdot \boldsymbol{n}^{I} d \cdot \mathscr{H} \mathscr{C}^{n-1}\right|=\left|Q_{0}\left(\partial_{*} I\right)\right| \leqq \eta(I) .
\end{gathered}
$$

From Theorem 5.3 it follows that $\boldsymbol{q}$ has divergence measure with $|\operatorname{div} \boldsymbol{q}| \leqq \eta$. Let $\hat{h}$ be associated with $\boldsymbol{q}$ according to Theorem 5.2. By Corollary 5.5, $\boldsymbol{q}$ induces a balanced Cauchy flux $Q$ defined on $\mathscr{S}_{\hat{h} \eta}$. By Proposition 4.5 there exists a full grid $G \subseteq G_{1}$ such that $\mathscr{G}_{G} \subseteq \mathscr{S}_{\hat{h} \eta}$. Then assertions (a) and (b) clearly follow.

Now assume also that $\check{Q}$ and $\check{\boldsymbol{q}}$ satisfy (a) for some $\check{G} \subseteq G_{0}$. If $G^{\prime}$ is a full grid with $G^{\prime} \subseteq G$ and $G^{\prime} \subseteq \check{G}$, we have

$$
Q(S)=\int_{S} \boldsymbol{q} \cdot \boldsymbol{n}_{S} d . \mathscr{H} \mathscr{C}^{n-1}=Q_{0}(S)=\check{Q}(S)=\int_{S} \check{\boldsymbol{q}} \cdot \boldsymbol{n}_{S} d . \mathscr{H} \mathscr{C}^{n-1}
$$

for any $S \in \mathscr{T}_{G^{\prime}}$. From Theorem 4.9 and Proposition 5.6 we conclude that $\check{Q}=Q$ on almost all of $\mathscr{S}$ and $\check{\boldsymbol{q}}(x)=\boldsymbol{q}(x)$ for $\mathscr{D}^{n}$-a.e. $x \in$ int $B$. 


\section{Integral Representation and Formulations of the Balance Law}

Let $(B, \mathscr{P})$ be a continuous body. The result of the previous section allows us to prove the converse of Corollary 5.5, which is one of the main goals of the paper.

Theorem 7.1. Let $Q$ be a balanced Cauchy flux on $(B, \mathscr{P})$. Then there exists a vector field $\boldsymbol{q} \in \mathscr{L}_{\text {loc }}^{1}$ (int $\left.B ; \mathbb{R}^{n}\right)$ with divergence measure such that

$$
Q(S)=\int_{S} \boldsymbol{q} \cdot \boldsymbol{n}_{S} d . \mathscr{H} \mathscr{C}^{n-1}
$$

on almost all of. $\mathscr{S}$. Moreover, $\boldsymbol{q}$ is uniquely determined $\mathscr{D}^{n}$-almost everywhere.

Proof. Let $h, \eta$ and $G$ be as in Theorem 4.6. Then the restriction of $Q$ to $\mathscr{T}_{G}$ satisfies the assumptions (i), (ii) and (iii) considered in the previous section. Let $Q^{\prime}, \boldsymbol{q}$ and $G^{\prime} \subseteq G$ be as in Theorem 6.1. Since $Q^{\prime}(S)=Q(S)$ for any $S \in \mathscr{S}_{G^{\prime}}$, we have $Q^{\prime}=Q$ on almost all of $\mathscr{S}$ by Theorem 4.9. Then the integral representation formula follows.

The uniqueness of $\boldsymbol{q}$ is a consequence of Corollary 5.7.

Moreover, we can prove the equivalence between the integral and the distributional formulation of the balance law. For the integral formulation, it turns out that it is enough to consider the elements of $\mathscr{T}_{G}$ for some full grid $G$.

Theorem 7.2. Let $\boldsymbol{q} \in \mathscr{D}_{\text {loc }}^{1}$ (int $B ; \mathbb{R}^{n}$ ), let $\mu \in \mathfrak{M}$ (int $B$ ) and let $u:$ int $B \rightarrow \mathbb{R}$ be a Borel function with $|u(x)|=1$ for $\mu$-a.e. $x \in$ int $B$. Then the following conditions are equivalent:

(a) For almost every $M \in \mathscr{P}, \boldsymbol{q}$ is $\mathscr{H} \mathfrak{C}^{n-1}$-summable on $\partial_{*} M$ and

$$
\int_{\partial_{*} M} \boldsymbol{q} \cdot \boldsymbol{n}^{M} d \cdot \mathscr{H}^{n-1}=\int_{M} u d \mu .
$$

(b) There exists a full grid $G$ such that $\mathbf{q}$ is $\mathscr{\mathscr { H }} \mathbb{C}^{n-1}$-summable on $\partial_{*} I$ and

$$
\int_{\partial_{*} I} \boldsymbol{q} \cdot \boldsymbol{n}^{I} d \cdot \mathscr{\mathscr { B } ^ { n - 1 }}=\int_{I} u d \mu \quad \text { for every } I \in \mathscr{T}_{G} .
$$

(c)

$$
-\int_{\text {int } B} \boldsymbol{q} \cdot \nabla f d \mathscr{B}^{n}=\int_{\text {int } B} f u d \mu \quad \text { for every } f \in C_{0}^{\infty} \text { (int } B \text { ). }
$$

Proof. (a) $\Longrightarrow$ (b). It follows from Proposition 4.5 .

(b) $\Longrightarrow$ (c). From Theorem 5.3 it follows that $\boldsymbol{q}$ has divergence measure. Let $h$ and $\eta$ be as in Theorem 5.4 and let $G_{1} \subseteq G$ be a full grid with $\mathscr{P}_{G_{1}} \subseteq \mathscr{P}_{h \mu} \cap \mathscr{P}_{h \eta}$. Then

$$
\int_{I} \operatorname{div} \boldsymbol{q}=\int_{\partial_{*} I} \boldsymbol{q} \cdot \boldsymbol{n}^{I} d \cdot \mathscr{\mathscr { B }} \mathscr{b}^{n-1}=\int_{I} u d \mu
$$


for any $I \in \mathscr{T}_{G_{1}}$. Since each open subset of int $B$ is a countable disjoint union of elements of $\mathscr{T}_{G_{1}}$ up to a set which is both $\mu$ - and $\eta$-negligible, we have

$$
\forall f \in C_{0}^{\infty}(\operatorname{int} B): \int_{\text {int } B} f \operatorname{div} \boldsymbol{q}=\int_{\text {int } B} f u d \mu
$$

and the assertion follows.

(c) $\Longrightarrow$ (a). This follows from Theorem 5.4.

\section{Symmetric Flux Tensors}

In this section we provide further information for vector Cauchy fluxes. We denote by $\operatorname{Lin}\left(\mathbb{R}^{n} ; \mathbb{R}^{N}\right)$ the normed space of linear maps from $\mathbb{R}^{n}$ to $\mathbb{R}^{N}$. If $L \in$ $\operatorname{Lin}\left(\mathbb{R}^{n} ; \mathbb{R}^{N}\right)$, then $L^{T} \in \operatorname{Lin}\left(\mathbb{R}^{N} ; \mathbb{R}^{n}\right)$ denotes its transpose. If $x \in \mathbb{R}^{n}$ and $y \in \mathbb{R}^{N}$, then $y \otimes x \in \operatorname{Lin}\left(\mathbb{R}^{n} ; \mathbb{R}^{N}\right)$ is defined by $(y \otimes x) z=(x \cdot z) y$. When $n=N$, we set $x \wedge y=x \otimes y-y \otimes x$.

Let $(B, \mathscr{P})$ be a continuous body with $B \subseteq \mathbb{R}^{n}$.

Definition 8.1. Let $\mathrm{q} \in \mathscr{L}_{\mathrm{loc}}^{1}\left(\operatorname{int} B ; \operatorname{Lin}\left(\mathbb{R}^{n} ; \mathbb{R}^{N}\right)\right)$. We say that div q is a (local vector) measure on int $B$, if div q is a (vector) distribution on int $B$ of order 0 . This means that for every compact subset $K$ of int $B$ there exists a constant $c_{K}$ such that

$$
\left|\int_{\operatorname{int} B} \mathrm{q} \nabla f d \mathscr{B}^{n}\right| \leqq c_{K} \max _{K}|f|
$$

whenever $f \in C_{0}^{\infty}$ (int $B$ ) and supt $f \subseteq K$. In such a case, there exist $\mu \in$ $\mathfrak{M}$ (int $B$ ) and a Borel map $\boldsymbol{u}:$ int $B \rightarrow \mathbb{R}^{N}$ such that $|\boldsymbol{u}(x)|=1$ for $\mu$-a.e. $x \in \operatorname{int} B$ and

$$
-\int_{\operatorname{int} B} \mathrm{q} \nabla f d \mathscr{B}^{n}=\int_{\operatorname{int} B} f \boldsymbol{u} d \mu
$$

for any Lipschitz function $f:$ int $B \rightarrow \mathbb{R}$ with compact support. It is well known that $\mu$ is uniquely determined, while $\boldsymbol{u}$ is uniquely determined $\mu$-almost everywhere. We put $|\operatorname{div} \mathrm{q}|=\mu$. Finally, if $M \in \mathfrak{B}$ (int $B$ ) and $\boldsymbol{v}: M \rightarrow \mathbb{R}^{N}$ is a Borel map such that $\boldsymbol{v} \wedge \boldsymbol{u}$ is $\mu$-summable on $M$, we set

$$
\int_{M} \boldsymbol{v} \wedge \operatorname{div} \mathrm{q}=\int_{M} \boldsymbol{v} \wedge \boldsymbol{u} d \mu
$$

Definition 3.9 can be easily adapted to vector balanced Cauchy fluxes $Q: \mathscr{D} \rightarrow$ $\mathbb{R}^{N}$. These fluxes are in natural correspondence with tensor fields $\mathrm{q} \in \mathscr{L}_{\operatorname{loc}}^{1}$ (int $B$; $\operatorname{Lin}\left(\mathbb{R}^{n} ; \mathbb{R}^{N}\right)$ ) with divergence measure. We shall not develop such details, as they are straightforward extensions of the results of the previous sections, but we shall study, in the case $n=N$, conditions under which the tensor $\mathrm{q}(x)$ is symmetric for $\mathscr{B}^{n}$-a.e. $x \in$ int $B$. 
Theorem 8.2. Let $\mathrm{q} \in \mathscr{D}_{\text {loc }}^{1}\left(\operatorname{int} B ; \operatorname{Lin}\left(\mathbb{R}^{n} ; \mathbb{R}^{N}\right)\right)$ be a tensor field with divergence measure and let $\eta=|\operatorname{div} q|$. Then there exists $h \in \mathscr{B}_{\mathrm{loc},+}^{1}($ int $B)$ such that, for every $M \in \mathscr{T h}$ and for every locally Lipschitz map $v:$ int $B \rightarrow \mathbb{R}^{N}$, the function $\mathrm{q}$ is $\mathscr{H}^{n-1}$-summable on $\partial_{*} M$ and

$$
\int_{M}\left((\nabla v) q^{T}-\mathbf{q}(\nabla v)^{T}\right) d \mathscr{D}^{n}=\int_{\partial_{*} M} \boldsymbol{v} \wedge\left(\mathbf{q} \boldsymbol{n}^{M}\right) d \mathscr{H} \mathscr{b}^{n-1}-\int_{M} \boldsymbol{v} \wedge \operatorname{div} \mathbf{q},
$$

where $\nabla \boldsymbol{v}(x) \in \operatorname{Lin}\left(\mathbb{R}^{n} ; \mathbb{R}^{N}\right)$ denotes the Fréchet differential of $\boldsymbol{v}$ at $x$.

Proof. It is sufficient to adapt Theorems 5.2 and 5.4.

Theorem 8.3. Let $\mathrm{q} \in \mathscr{D}_{\text {loc }}^{1}$ (int $\left.B ; \operatorname{Lin}\left(\mathbb{R}^{n} ; \mathbb{R}^{n}\right)\right)$ be a tensor field with divergence measure. Then the following conditions are equivalent:

(a) For each $x_{0} \in \mathbb{R}^{n}$ and almost every $M \in \mathscr{P}$, $\mathrm{q}$ is $\mathscr{\mathscr { B }}{ }^{n-1}$-summable on $\partial_{*} M$ and

$$
\int_{\partial_{*} M}\left(x-x_{0}\right) \wedge\left(\mathrm{q}(x) n^{M}(x)\right) d \mathscr{H}^{n-1}(x)=\int_{M}\left(x-x_{0}\right) \wedge \operatorname{div} \mathrm{q}(x) .
$$

(b) There exists $\eta \in \mathfrak{M}$ (int $B$ ) such that, for each $x_{0} \in \mathbb{R}^{n}$ and almost every $M \in \mathscr{P}$, q is $\mathscr{H}^{n-1}$-summable on $\partial_{*} M$ and

$$
\left|\int_{\partial_{*} M}\left(x-x_{0}\right) \wedge\left(\mathrm{q}(x) \boldsymbol{n}^{M}(x)\right) d \mathscr{H}^{n-1}(x)\right| \leqq\left(\sup _{x \in M}\left|x-x_{0}\right|\right) \eta(M) .
$$

(c) There exist $\eta \in \mathfrak{M}$ (int $B$ ) and a full grid $G$ such that, for every $x_{0} \in \mathbb{R}^{n}$ and every $I \in \mathscr{T}_{G}$, q is $\mathscr{H} \mathscr{C}^{n-1}$-summable on $\partial_{*} I$ and

$$
\left|\int_{\partial_{*} I}\left(x-x_{0}\right) \wedge\left(\mathrm{q}(x) \boldsymbol{n}^{I}(x)\right) d \cdot \mathscr{H}^{n-1}(x)\right| \leqq\left(\sup _{x \in I}\left|x-x_{0}\right|\right) \eta(I) .
$$

(d) For $\mathscr{D}^{n}$-a.e. $x \in$ int $B$ there exists a sequence $\left(I_{m}\right)$ of open $n$-dimensional intervals such that $x \in I_{m}, \lim _{m}\left(\operatorname{diam} I_{m}\right)=0$, q is. $\mathscr{H}^{n-1}$-summable on $\partial_{*} I_{m}$ and

$$
\begin{gathered}
\limsup _{m} \frac{\left(\operatorname{diam} I_{m}\right)^{n}}{\mathscr{B}^{n}\left(I_{m}\right)}<+\infty \\
\lim _{m} \frac{\int_{\partial_{*} I_{m}}(\xi-x) \wedge\left(\mathrm{q}(\xi) n^{I_{m}}(\xi)\right) d \mathscr{H}^{n-1}(\xi)}{\mathscr{D}^{n}\left(I_{m}\right)}=0 .
\end{gathered}
$$

(e) $\mathrm{q}(x)$ is symmetric for $\mathscr{B}^{n}$-a.e. $x \in$ int $B$.

Proof. $(\mathrm{a}) \Longrightarrow(\mathrm{b})$. We have

$$
\left|\int_{M}\left(x-x_{0}\right) \wedge \operatorname{div} \mathrm{q}(x)\right| \leqq\left(\sup _{x \in M}\left|x-x_{0}\right|\right)|\operatorname{div} \mathrm{q}|(M)
$$

whence the assertion.

(b) $\Longrightarrow$ (c). This follows from Proposition 4.5 . 
(c) $\Longrightarrow($ d). Let $x \in$ int $B$ be such that

$$
\limsup _{r \rightarrow 0^{+}} \frac{\eta\left(\mathrm{B}_{r}(x)\right)}{\mathscr{D}^{n}\left(\mathrm{~B}_{r}(x)\right)}<+\infty .
$$

It is well known that $\mathscr{B}^{n}-$ a.e. $x \in \operatorname{int} B$ has this property. Moreover, let $\left(I_{m}\right)$ be a sequence in $\mathscr{T}_{G}$ such that $x \in I_{m}, \lim _{m}\left(\operatorname{diam} I_{m}\right)=0$ and

$$
\limsup _{m} \frac{\left(\operatorname{diam} I_{m}\right)^{n}}{\mathscr{B}^{n}\left(I_{m}\right)}<+\infty .
$$

Since $I_{m} \subseteq \mathrm{B}_{\operatorname{diam} I_{m}}(x)$ and $\left(\operatorname{diam} I_{m}\right)^{n} \leqq c \mathscr{B}^{n}\left(I_{m}\right)$ for some constant $c$, it is readily seen that

$$
\underset{m}{\limsup } \frac{\eta\left(I_{m}\right)}{\mathscr{D}^{n}\left(I_{m}\right)}<+\infty
$$

whence the assertion.

$(\mathrm{d}) \Longrightarrow(\mathrm{e})$. Let $x \in \operatorname{int} B$ and $\left(I_{m}\right)$ be as in assertion $(d)$. Without loss of generality, we may assume that

$$
\begin{gathered}
\limsup _{r \rightarrow 0^{+}} \frac{|\operatorname{div} \mathrm{q}|\left(\mathrm{B}_{r}(x)\right)}{\mathscr{D}^{n}\left(\mathrm{~B}_{r}(x)\right)}<+\infty, \\
\lim _{r \rightarrow 0^{+}} \frac{1}{\mathscr{L}^{n}\left(\mathrm{~B}_{r}(x)\right)} \int_{\mathrm{B}_{r}(x)}|\mathrm{q}(\xi)-\mathrm{q}(x)| d \mathscr{B}^{n}(\xi)=0 .
\end{gathered}
$$

Then it is readily seen that

$$
\begin{gathered}
\limsup _{m} \frac{|\operatorname{div} \mathrm{q}|\left(I_{m}\right)}{\mathscr{D}^{n}\left(I_{m}\right)}<+\infty, \\
\lim _{m} \frac{1}{\mathscr{S}^{n}\left(I_{m}\right)} \int_{I_{m}}|\mathrm{q}(\xi)-\mathrm{q}(x)| d \mathscr{B}^{n}(\xi)=0 .
\end{gathered}
$$

On the other hand, by Theorem 8.2 we have

$$
\begin{aligned}
\int_{I_{m}}\left(\mathrm{q}(\xi)^{T}-\mathrm{q}(\xi)\right) d \mathscr{B}^{n}(\xi) \\
\quad=\int_{\partial_{*} I_{m}}(\xi-x) \wedge\left(\mathrm{q}(\xi) \boldsymbol{n}^{I_{m}}(\xi)\right) d \cdot \mathscr{H}^{n-1}(\xi)-\int_{I_{m}}(\xi-x) \wedge \operatorname{div} \mathrm{q}(\xi),
\end{aligned}
$$

whence

$$
\begin{aligned}
& \left|\int_{I_{m}}\left(\mathrm{q}(\xi)^{T}-\mathrm{q}(\xi)\right) d \mathscr{B}^{n}(\xi)\right| \\
& \quad \leqq\left|\int_{\partial_{*} I_{m}}(\xi-x) \wedge\left(\mathrm{q}(\xi) \boldsymbol{n}^{I_{m}}(\xi)\right) d \mathscr{H}^{n-1}(\xi)\right|+\left(\operatorname{diam} I_{m}\right)|\operatorname{div} \mathrm{q}|\left(I_{m}\right) .
\end{aligned}
$$

If we divide both sides by $\mathscr{D}^{n}\left(I_{m}\right)$ and we pass to the limit as $m \rightarrow \infty$, we get $\mathrm{q}(x)^{T}-\mathrm{q}(x)=0$, whence the assertion.

(e) $\Longrightarrow$ (a). It is sufficient to apply Theorem 8.2 with $\boldsymbol{v}(x)=x-x_{0}$. 
Acknowledgements. We thank CARLO BANFI for suggesting the study of tensor fields with divergence measure, and for his encouragement. The research of M. DEGIOvanNI was partially supported by Ministero dell'Università e della Ricerca Scientifica e Tecnologica (40\% - 1995) and by Gruppo Nazionale per l'Analisi Funzionale e le sue Applicazioni. The research of A. MARZOCCHI was partially supported by Ministero dell'Università e della Ricerca Scientifica e Tecnologica (40\% - 1995) and by Gruppo Nazionale per la Fisica Matematica.

\section{References}

1. S. S. Antman \& J. E. Osborn, The principle of virtual work and integral laws of motion, Arch. Rational Mech. Anal. 69 (1979), 231-262.

2. C. BANFi \& M. FABrizio, Sul concetto di sottocorpo nella meccanica dei continui, Atti Accad. Naz. Lincei Rend. Cl. Sci. Fis. Mat. Natur. (8) 66 (1979), 136-142.

3. H. Brezis, Analyse Fonctionnelle. Théorie et Applications, Masson, Paris, 1983.

4. H. Federer, Geometric Measure Theory, Springer-Verlag, New York, 1969.

5. W. Fleming, Functions of Several Variables, Springer-Verlag, New York Heidelberg, 1977.

6. R. L. FosDick \& E. G. Virga, A variational proof of the stress theorem of Cauchy, Arch. Rational Mech. Anal. 105 (1989), 95-103.

7. E. GiUsti, Minimal Surfaces and Functions of Bounded Variation, Birkhäuser, Boston, 1984.

8. M. E. Gurtin \& L. C. Martins, Cauchy's theorem in classical physics, Arch. Rational Mech. Anal. 60 (1976), 306-324.

9. M. E. Gurtin, V. J. Mizel \& W. O. Williams, A note on Cauchy's stress theorem, $J$. Math. Anal. Appl. 22 (1968), 398-401.

10. M. E. Gurtin \& W. O. Williams, An axiomatic foundation for continuum thermodynamics, Arch. Rational Mech. Anal. 26 (1967), 83-117.

11. M. E. Gurtin, W. O. Williams \& W. P. Ziemer, Geometric measure theory and the axioms of continuum thermodynamics, Arch. Rational Mech. Anal. 92 (1986), 1-22.

12. W. NolL, The foundations of classical mechanics in the light of recent advances in continuum mechanics, in The Axiomatic Method, with Special Reference to Geometry and Physics (Berkeley, 1957/58), 266-281, Studies in Logic and the Foundations of Mathematics, North-Holland, Amsterdam, 1959.

13. W. Noll, Lectures on the foundations of continuum mechanics, Arch. Rational Mech. Anal. 52 (1973), 62-92.

14. W. Noll \& E. G. VIRGA, Fit regions and functions of bounded variation, Arch. Rational Mech. Anal. 102 (1988), 1-21.

15. M. ŚlLhaVÝ, The existence of the flux vector and the divergence theorem for general Cauchy fluxes, Arch. Rational Mech. Anal. 90 (1985), 195-211.

16. M. Š ILHAVÝ, Cauchy's stress theorem and tensor fields with divergences in $L^{p}$, Arch. Rational Mech. Anal. 116 (1991), 223-255.

17. L. M. Simon, Lectures on Geometric Measure Theory, Proceedings of the Centre for Mathematical Analysis, Australian National University, 3, Canberra, 1983.

18. C. A. Truesdell, A First Course in Rational Continuum Mechanics, Vol. 1, Academic Press, Boston, 1991.

19. W. P. Ziemer, Cauchy flux and sets of finite perimeter, Arch. Rational Mech. Anal. 84 (1983), 189-201.

20. W. P. Ziemer, Weakly Differentiable Functions, Springer-Verlag, New York, 1989.

Dipartimento di Matematica e Fisica

Università Cattolica del Sacro Cuore

Via Trieste 17

I-25121 Brescia, Italy 